\title{
Engineered Exosomes Derived From Primary M2 Macrophages With Anti-inflammatory And Neuroprotective Properties For The Treatment of Spinal Cord Injury.
}

\section{Chuanjie Zhang}

First Affiliated Hospital of Jinzhou Medical University

\section{Daoyong Li}

First Affiliated Hospital of Jinzhou Medical University

Hengshuo Hu

First Affiliated Hospital of Jinzhou Medical University

\section{Zhe Wang}

First Affiliated Hospital of Jinzhou Medical University

Jinyu An

Jinzhou Medical University

\section{Zhanshan Gao}

Jinzhou Medical University

\section{Kaihua Zhang}

First Affiliated Hospital of Jinzhou Medical University

Xifan Mei ( $\nabla$ meixifan@jzmu.edu.cn )

First Affiliated Hospital of Jinzhou Medical University https://orcid.org/0000-0003-3698-0525

Chao Wu

Jinzhou Medical University

He Tian

Jinzhou Medical University

\section{Research Article}

Keywords: Spinal cord injury, Exosomes, Curcumin, M2 re-polarization, Neuroprotection

Posted Date: July 27th, 2021

DOI: https://doi.org/10.21203/rs.3.rs-721536/v1

License: (9) This work is licensed under a Creative Commons Attribution 4.0 International License. Read Full License 
Page $2 / 28$ 


\section{Abstract}

Background: Uncontrollable inflammation and nerve cell apoptosis are the most destructive pathological response after spinal cord injury $(\mathrm{SCl})$. So, inflammation suppression combined with neuroprotection is one of the most promising strategies to treat SCl. Engineered exosomes with anti-inflammatory and neuroprotective properties are promising candidates for the implementation of this strategies for the treatment of SCl.

Results: By combining nerve growth factor (NGF) and curcumin (Cur), we prepared stable engineered exosomes of approximately $120 \mathrm{~nm}$ from primary M2 macrophages with anti-inflammatory and neuroprotective properties (Cur@EXs $\mathrm{s}^{-\mathrm{Cl}-\mathrm{NGF}}$ ). Notably, NGF was coupled with EXs by matrix metalloproteinase 9 (MMP9)-cleavable linker to accurately release at the injured site. Through targeted experiments, we found that these exosomes could actively and effectively accumulate at the injured site of SCI mice, which greatly improved the bioavailability of the drugs. Subsequently, Cur@EXs-cl-NGF reached the injured site and could effectively inhibit the uncontrollable inflammatory response to protect the spinal cord from secondary damage; in addition, Cur@EXs ${ }^{\text {-lI-NGF }}$ could release NGF into the microenvironment in time to exert a neuroprotective effect against nerve cell damage.

Conclusions: A series of in vivo and in vitro experiments showed that the engineered exosomes significantly improved the microenvironment after injury and promoted the recovery of motor function after $\mathrm{SCl}$. We provide a new method for inflammation suppression combined with neuroprotective strategies to treat $\mathrm{SCl}$.

\section{Background}

Spinal cord injury (SCl), associated with complicated pathological changes and irreversible nerve damage, is one of the most destructive diseases [1]. Patients often experience a permanent loss of motor and sensory functions, leading to a decreased quality of life and increased economic burden on the family and society [2]. SCl is characterized both by primary injury caused by acute and focal lesions (including destruction of the vasculature and the blood-spinal cord barrier) and extensive secondary injury composed of ischemia, hypoxia, and uncontrolled neuroinflammatory reactions $[3,4]$. Antiinflammatory and neuroprotective strategies are still the starting point of current $\mathrm{SCl}$ drug treatment $[5,6]$. Therefore, anti-inflammatory drugs and neurotrophic drugs are usually included in the treatment regimen for SCl [7]. However, due to the short half-life, low solubility, and low accumulation at the injured site, it is difficult for clinical medications to exert satisfactory anti-inflammatory and neurotrophic effects [8].

In recent years, exosomes, as a new type of drug carrier, have attracted widespread attention from researchers [9]. Increasing numbers of studies have shown that exosomes, with obvious advantages of high biocompatibility, low cytotoxicity, immune inertia, and long-term circulation, have broad application prospects in improving drug delivery and therapeutic effects $[10,11]$. M2 macrophages play a crucial inhibitory effect on inflammation in various inflammatory diseases, including $\mathrm{SCl}$ [12]. Interestingly, 
exosomes derived from M2 macrophages (EXs) can inherit diverse anti-inflammatory cytokines, such as interleukin-10 (IL-10) and transforming growth factor (TGF- $\beta$ ), as well as various chemokine receptors from the macrophage surface, such as chemokine receptor type 2 (CCR2) [13]. More significantly, EXs have played a central role in the treatment of inflammatory diseases, such as atherosclerosis, skin damage and stroke $[13,14]$. Thus, EXs with both inflammation suppression and targeting functions are ideal drug carriers. Previous research by our team has confirmed that EXs carrying berberine can effectively reduce the inflammatory response after $\mathrm{SCl}$ to promote the recovery of motor function [15].

Nerve growth factors (NGF) can reduce neuronal apoptosis, promote angiogenesis, and promote axon extension and functional recovery $[16,17]$. After $\mathrm{SCl}$, hypoxia-ischemia leads to a significant decrease in endogenous expression of NGF at the injury site, highlighting the importance of delivering exogenous NGF $[18,19]$. However, previous research has indicated that the biological half-life of NGF after intravenous injection is approximately $2.3 \mathrm{~h}$, so poor bioavailability greatly limits the accumulation of NGF in target cells [20]. To prolong the circulation time of NGF in the body, we coupled NGF to the surface of EXs by the matrix metalloproteinase 9 (MMP9) cleavable linker (cl), which had a sequence of RVGLP, to prepare EXs ${ }^{-{ }^{-N}-N G F}[21,22]$. Considering that MMP9 expression is significantly increased at the injury site after SCl, NGF in EXs ${ }^{-\mathrm{Cl}-\mathrm{NGF}}$ can dissociate from EXs and be released into the microenvironment over time $[23,24]$. The NGF is then taken up by nerve cells through NGF receptor-mediated endocytosis, avoiding consumption by inflammatory cells [25].

Curcumin (Cur) is a natural polyphenol with excellent anti-inflammatory properties that promotes the polarization of M1 macrophages to M2 macrophages [26, 27]. Simultaneously, a low solubility, poor stability, low absorption rate and short biological half-life lead to its low bioavailability, which limits its anti-inflammatory application in vivo $[28,29]$. To overcome these shortcomings, Cur was loaded into EXs ${ }^{-\mathrm{cl}-N G F}$, and Cur@EXs ${ }^{-\mathrm{cl}-\mathrm{NGF}}$ was prepared for the treatment of SCl.

In this work, the designed Cur@EXs ${ }^{-\mathrm{Cl}-\mathrm{NGF}}$ had both anti-inflammatory and neuroprotective properties. The design concept and mechanism of action are shown in the schematic diagram below (Figure. 1). These effects were verified in vitro using cell experiments and in vivo using animal experiments. We hope that this design can provide new ideas for the clinical treatment of $\mathrm{SCl}$.

\section{Results}

\section{Preparation and characterization of EXs ${ }^{-\mathrm{cl}-\mathrm{NGF}}$.}

After IL-4 $(20 \mathrm{ng} / \mathrm{mL})$ stimulation for $48 \mathrm{~h}$, flow cytometry showed that approximately $85.03 \%$ of primary macrophages obtained from the abdominal cavity of mice had differentiated into M2 macrophages with anti-inflammatory effects (Figure. 2A). Next, we used western blotting to detect the expression of M2 macrophage marker proteins (ARG-1 and CD206). The results further verified that M2 macrophages were induced successfully (Figure. 2B). Transmission electron microscopy (TEM) showed the classic morphology of EXs with an average diameter of $\sim 120 \mathrm{~nm}$ (Figure. 2C, Figure. 2D). Then, the successful 
connection of NGF and EXs through a metalloproteinase 9 (MMP9) cleavable linker was verified by the binding of secondary antibody-linked Au nanoparticles (Figure. 2E). The high degree of colocalization of NGF and EXs under CLSM and STED further validated the success of the modification (Figure. 2F). The proportion of colocalization was approximately $89.72 \%$ (Figure. $2 \mathrm{G}$ ). Nanoparticle tracking analysis (NTA) showed that after modification with NGF, the size of EXs ${ }^{-\mathrm{Cl}-\mathrm{NGF}}$ was slightly increased compared with that of EXs, while the zeta potential was significantly reduced to $33.56 \mathrm{mV}$ from $42.17 \mathrm{mV}$ (Figure. $2 \mathrm{H}$ ). Western blotting was used to detect the expression of exosome marker proteins (CD9 and TSG101), chemokine receptors (CCR2) mediating the chemotaxis of exosomes to inflammatory lesions, and inflammation inhibitory cytokines (IL-10) in EXs and EXs ${ }^{-\mathrm{Cl}-\mathrm{NGF}}$. We found that expression of the marker protein and functional protein of the modified EXs did not change significantly (Figure. 2I). Based on ELISA kits, we detected the release ability of NGF from EXs ${ }^{-{ }^{-1-N G F}}$. After adding MMP9 to the PBS solution of EXs ${ }^{-\mathrm{Cl}-\mathrm{NGF}}$, the NGF linked on the surface of EXs was effectively dissociated. The solution with MMP9 and the MMP9 inhibitor GM6001 together did not dissociate NGF, which showed that the cleavable linker was specific to MMP9 (Figure. 2J). Subsequently, the PC12 cells growth rate was determined with an MTT assay. Comparing the NGF released from EXs ${ }^{-{ }^{-C l}-N G F}$ with the free form of NGF, we found no significant difference between free NGF and NGF in EXs ${ }^{-\mathrm{Cl}-\mathrm{NGF}}$ in promoting the proliferation of PC12 cells. This result indicated that the binding and release of NGF did not damage the

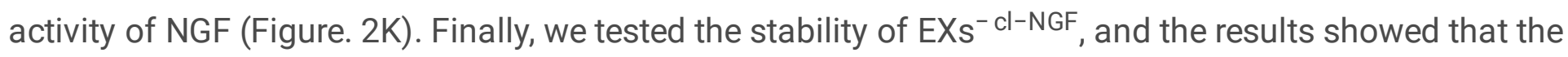
size (Figure. $2 \mathrm{~L}$ ) and zeta potential (Figure. $2 \mathrm{M}$ ) of EXs ${ }^{-\mathrm{Cl}-\mathrm{NGF}}$ did not change significantly after storage in PBS at $4^{\circ} \mathrm{C}$ for $7 \mathrm{~d}$. Moreover, after lyophilization and reconstitution, the size and zeta potential of EXs ${ }^{-}$ cl-NGF changed only slightly. (Figure. 2N, Figure. 20). These results indicated that EXs ${ }^{-\mathrm{cl}^{-N G F}}$ had the stability needed for future clinical applications.

\section{The ability of EXs ${ }^{-\mathrm{cl}-\mathrm{NGF}}$ to target sites of inflammation and release NGF.}

To evaluate whether EXs ${ }^{-\mathrm{Cl}-\mathrm{NGF}}$, as a carrier of NGF, could effectively deliver NGF to the injured site, we used a living imaging system to detect the inflammation targeting ability of $\mathrm{EXs}^{-\mathrm{Cl}-\mathrm{NGF}}$ in $\mathrm{SCl}$ animal models. After $\mathrm{SCl}$, the mice were randomly divided into 3 groups, NGF, RVs ${ }^{-\mathrm{Cl}-\mathrm{NGF}}$, or EXs ${ }^{-\mathrm{cl}-\mathrm{NGF}}$ (NGF in each group was labeled with $\mathrm{CY} 7$ fluorescent dye), and different nanoparticles were injected through the tail vein. A living imaging system showed that the free form of NGF hardly accumulated at the injury site, and $\mathrm{RVs}^{-}{ }^{-\mathrm{Cl}-\mathrm{NGF}}$ could exist at the injury site for a shorter time owing to the passive targeting ability of the red blood cell membrane nanoparticles. However, EXs ${ }^{-{ }^{-C}-N G F}$ exhibited the strongest fluorescence intensity and the longest accumulation time at the injury site, demonstrating the excellent ability of EXs ${ }^{-}$ cl-NGF to target inflammation (Figure. 3A). Quantitative statistics of the fluorescence image demonstrated that NGF delivered by EXs ${ }^{-\mathrm{Cl}-\mathrm{NGF}}$ accumulated at the injured site within $2 \mathrm{~h}$ after injection, reached a peak at approximately $12 \mathrm{~h}$ after injection and persisted for more than $72 \mathrm{~h}$ (Figure. 3B). Fluorescence images of the main organs (heart, liver, spleen, lung and kidney) and spinal cord from different groups were collected $12 \mathrm{~h}$ after tail vein injection, further indicating the satisfactory targeting ability of EXs ${ }^{-\mathrm{cl}^{-N G F}}$ for the injured spinal cord (Figure. $3 \mathrm{C}$ ). Statistical analysis showed that the fluorescence intensity of EXs ${ }^{-}$ 
cl-NGF was approximately eight times that of free NGF in the spinal cord, while the kidneys of mice injected with free NGF had obvious fluorescence accumulation (Figure. 3D). An NGF ELISA kit was used to detect the biological half-life of NGF in the blood of each group. The results showed that the circulation time in the body of NGF in EXs ${ }^{-\mathrm{Cl}-\mathrm{NGF}}$ was significantly prolonged due to the long-term circulating ability of EXs (Figure. 3E). Next, we constructed the Transwell ${ }^{\text {TM }}$ coculture system to verify whether the cleavable linker contributed to the uptake of NGF by nerve cells. The upper layer of the Transwell ${ }^{\text {TM }}$ coculture system consisted of PC12 cells, and the lower layer was primary M1 macrophages. Then, we added free NGF, $\mathrm{EXs}^{-\mathrm{NGF}}$, or EXs ${ }^{-\mathrm{cl}-\mathrm{NGF}}$ (NGF in each group was labeled with FITC) to the Transwell ${ }^{\text {TM }}$ coculture system. The schematic diagram is shown (Figure. 3F, Figure. 3K). CLSM images showed that the fluorescence intensity of PC12 cells was significantly lower in the EXs ${ }^{-N G F}$ group than in the groups free NGF and $\mathrm{EXs}^{-\mathrm{Cl}-\mathrm{NGF}}$ (Figure. $3 \mathrm{G}$ ), while the fluorescence intensity of M1 macrophages cultured in the lower layer was significantly higher than that in the groups free NGF and EXs ${ }^{-\mathrm{Cl}-\mathrm{NGF}}$ (Figure. 3L). Fluorescence intensity statistical analysis further demonstrated that compared with the EXs ${ }^{-N G F}$ group, the amount of NGF endocytosis increased significantly in PC12 cells in the EXs ${ }^{- \text {Cl-NGF }}$ group (Figure. 3l) but was obviously reduced in $\mathrm{M} 1$ macrophages (Figure. $3 \mathrm{~N}$ ). This finding suggested that due to the presence of a cleavable linker, NGF of EXs ${ }^{-}$cl-NGF could be released into the microenvironment to be taken up by PC12 cells in time to reduce the consumption of NGF by M1 macrophages. Subsequently, we used flow cytometry to verify the endocytosis of NGF in different groups over time in PC12 cells or M1 macrophages (Figure. 3H, Figure. 3M). Quantitative analysis of flow cytometry indicated that NGF from EXs ${ }^{-\mathrm{Cl}-N G F}$ and free NGF had a faster rate of endocytosis and greater uptake by PC12 cells (Figure. $3 \mathrm{~J}$ ), while M1 macrophages showed very little uptake (Figure. 30).

\section{In vitro inflammation regulation and neuroprotective capabilities of EXs $\mathrm{sl}^{-\mathrm{C} G F}$}

To test the anti-inflammatory and neuroprotective capabilities of EXs ${ }^{-\mathrm{cl}^{-N G F}}$, we conducted Transwell ${ }^{\mathrm{TM}}$ coculture tests. Briefly, primary macrophages were induced to differentiate into M1 macrophages with LPS for $24 \mathrm{~h}$ and then cultured in the lower layer of the system. After $\mathrm{H}_{2} \mathrm{O}_{2}$ pretreatment for $12 \mathrm{~h}$ to simulate oxidative stress after $\mathrm{SCl}, \mathrm{PC} 12$ cells were cultured in the upper layer of the system. After the addition of different nanoparticles to the system for $12 \mathrm{~h}$, we evaluated the survival rate of PC12 cells and the degree of inflammation inhibition in each group. Flow cytometry (Figure. 4A) and live/dead cell staining (Figure. 4B) were used to detect the survival rate of PC12 cells under various treatment conditions. The flow cytometry results showed that under the action of EXs ${ }^{-{ }^{-N}-\mathrm{NGF}}$, the apoptotic rate of PC12 cells decreased from 41.53-12.74\%. The statistical results of live/dead cell staining further confirmed the neuroprotective effect of EXs ${ }^{-{ }^{-1-N G F}}$. Notably, compared with the PBS group, the survival rate of PC12 cells in the EXs group was also significantly improved, which suggested that inhibiting the inflammatory response in the system might be beneficial to the survival of injured nerve cells.

Simultaneously, based on ELISA kits, we detected the expression of proinflammatory factors (TNF-a, IL$1 \beta$, and IL-6) and anti-inflammatory factors (TGF- $\beta$ ) in the supernatants (Figure. 4C). As expected, in the EXs, EXs ${ }^{-N G F}$, and EXs ${ }^{-{ }^{-N}-N G F}$ groups under the action of EXs, the expression of proinflammatory factors 
(TNF-a, IL-1 $\beta$, and IL-6) was significantly reduced, and the expression of anti-inflammatory factors (TGF$\beta$ ) was obviously increased. Flow cytometry detection of the macrophage phenotype showed that the proportion of M1 macrophage subpopulations (CD86+) dropped to $49.79 \%$ from $91.16 \%$ in the EXs ${ }^{- \text {cl-NGF }^{-N}}$ group, suggesting that LPS induction was significantly blunted (Figure. 4D). Moreover, the proportion of M2 macrophage subpopulations (CD206+) rose to $10.27 \%$ from $1.59 \%$ (Figure. 4E). It was rather remarkable that the EXs group and EXs ${ }^{-N G F}$ group also had obvious inflammation inhibitory effects under the action of EXs.

\section{Cur@EXs ${ }^{-\mathrm{cl}-N G F}$ showed better anti-inflammatory effects via Cur delivery.}

As a small molecule drug, although Cur has excellent anti-inflammatory and antioxidant functions, its poor solubility and low bioavailability limit its effectiveness in vivo [27]. Therefore, we opted for Cur as a model drug of small molecule drugs to enhance the anti-inflammatory function of EXs ${ }^{-\mathrm{cl}-\mathrm{NGF}}$. The process of loading Cur into EXs ${ }^{-\mathrm{Cl}-\mathrm{NGF}}$ by the ultrasonic method is shown in the schematic diagram (Figure. 5A). We calculated that the percent drug load is about $37.68 \%$. CLSM and STED were used to detect whether Cur was successfully loaded into EXs ${ }^{-{ }^{-N}-N G F}$. The results showed that NGF, EXs, and Cur presented a high degree of colocalization, indicating the success of the loading (Figure. 5B). Conducting the drug release test in vitro, we found that Cur in Cur@EXs ${ }^{-\mathrm{Cl}-\mathrm{NGF}}$ showed slow release behavior in vitro. At $48 \mathrm{~h}$, the cumulative release reached approximately 78.84\%. (Figure. 5C). Then, we used flow cytometry to examine the effect of Cur@EXs ${ }^{-\mathrm{Cl}-\mathrm{NGF}}$ on the repolarization of M1 macrophages located in the lower layer of the Transwell ${ }^{\text {TM }}$ coculture system. It was rather remarkable that compared with the $\mathrm{EXs}^{-}$ cl-NGF, the ratio of M1 macrophage subpopulations (CD86+) was reduced in the Cur@EX ${ }^{-\mathrm{cl}-\mathrm{NGF}}$ group to $26.90 \%$ (Figure. 5D), and the ratio of M2 macrophage subpopulations (CD206+) also increased to $31.77 \%$ (Figure. 5E). This result suggested that by loading Cur, Cur@EXs ${ }^{-\mathrm{Cl}-\mathrm{NGF}}$ achieved a better antiinflammatory effect, which would be more suitable for the regulation of inflammatory diseases than EXs ${ }^{-}$ cl-NGF. In addition, ELISA kits were used to detect the levels of proinflammatory factors (TNF- $a$, IL-1 $\beta$, and IL-6) and anti-inflammatory factors (TGF- $\beta$ ) in the supernatant. As expected, under the combined effect of Cur and EXs, the Cur@EXs ${ }^{\text {- Cl-NGF }}$ group showed the best anti-inflammatory effect (Figure. 5F). To more intuitively observe the changes in macrophage polarization-related proteins (iNOS, ARG-1) at the cellular level between the groups, we performed immunofluorescence staining on primary macrophages in the lower layer of the TranswellTM. The CLSM images showed that, compared with the PBS group, the expression of marker protein (iNOS) of M1 macrophage subpopulations was significantly reduced in the EXs $^{-{ }^{-C l-N G F}}$ group and Cur@EXs ${ }^{-{ }^{-1-N G F}}$ group, and the expression of marker protein (ARG-1) of M2 macrophage subpopulations was significantly increased (Figure. 5G). Subsequently, fluorescence quantitative statistical analysis showed that the expression of iNOS in the Cur@EXs ${ }^{-\mathrm{cl}-\mathrm{NGF}}$ group was obviously lower than that in the EXs ${ }^{-\mathrm{Cl}-\mathrm{NGF}}$ group (Figure. $5 \mathrm{H}$ ). Moreover, the expression of ARG-1 showed the opposite result to iNOS, which further confirmed that Cur@EXs ${ }^{-{ }^{-N}-N G F}$ had the strongest regulatory effect on macrophage polarization (Figure. 5I). 


\section{Cur@EXs ${ }^{-c l-N G F}$ promotes the functional recovery of mice with SCl.}

To explore the therapeutic effect of Cur@EXs ${ }^{-\mathrm{Cl}-\mathrm{NGF}}$, mice with $\mathrm{SCl}$ were randomly divided into 5 groups: (1) Sham, (2) Normal saline (NS), (3) Cur, (4) EXs ${ }^{-\mathrm{Cl}-N G F}$, and (5) Cur@EXs ${ }^{-\mathrm{cl}-N G F}$. Since the inflammatory reaction occurs immediately and increases significantly within 3-6 h after the injury [30,31], we started administering treatment within 2-3 h after SCl. We selected $2 \mathrm{~h}, 2 \mathrm{~d}, 4 \mathrm{~d}$, and $6 \mathrm{~d}$ after injury as the administration time, and the mice were sacrificed at $28 \mathrm{~d}$ after injury to evaluate the recovery status of each group. First, we assessed the ultrastructure of myelin sheaths of mice that received different treatments by TEM (Figure. 6A). The photo showed that the myelin sheaths in the sham group were arranged in a tight and order manner, the thickness was uniform, and the layered structure was clear. The ultrastructure of myelin sheaths in the mice treated with NS was the most severely damaged. However, after Cur treatment, the amelioration of myelin sheaths was very limited. After EXs ${ }^{-\mathrm{Cl}-\mathrm{NGF}}$ and Cur@EXs ${ }^{-}$ cl-NGF treatments, the numbers, diameters and thickness of the myelin sheaths were significantly improved. Specifically, the protective effect of Cur@EXs ${ }^{-c^{-N G F}}$ on myelin sheaths was superior. The spinal cord from each group of mice was subjected to H\&E staining to further investigate the lesion changes at the injury site (Figure. 6B). The results showed that compared with the NS treatment group, the spinal cord in the Cur@EXs ${ }^{-\mathrm{Cl}-\mathrm{NGF}}$ treatment group was significantly more complete, its lesions were significantly reduced, and pathological changes were significantly improved. Moreover, both the number of surviving neuronal axon tubulin and the severity of scars caused by astrocytes were closely related to the recovery of motor function in injured mice. Therefore, we observed differences in the expression of GFAP and $\beta 3$-Tubulin in each group of mice at $28 \mathrm{~d}$ after injury by immunofluorescence staining (Figure. $6 \mathrm{C})$. The statistical analysis of $\beta 3$-Tubulin fluorescence showed that the number of surviving microtubule axon proteins in the EXs ${ }^{-\mathrm{Cl}-\mathrm{NGF}}$ and Cur@EXs ${ }^{-\mathrm{Cl}-\mathrm{NGF}}$ groups was significantly higher than that in the NS groups and Cur groups; in particular, the Cur@EXs ${ }^{-\mathrm{Cl}-\mathrm{NGF}}$ group had the strongest expression of $\beta 3$ Tubulin (Figure. 6D). The fluorescence statistics of GFAP showed that scar formation in the EXs ${ }^{-{ }^{-C l}-N G F}$ and Cur@EXs ${ }^{-\mathrm{Cl}-\mathrm{NGF}}$ groups was significantly less than that in the NS and Cur groups (Figure.6D). These results further confirmed the excellent therapeutic effect of Cur@EXs ${ }^{-\mathrm{Cl}-\mathrm{NGF}}$ on mice with SCI. Finally, we evaluated the effects of different treatments on motor function recovery through behavioral analysis. Footprint behavioral assays showed that in the sham group with a steady stride, the hind foot prints were clearly on the ground, the forefoot stride length was the longest and the stride width was the narrowest (Figure. 6E). In contrast, the footprints of the NS group were the poorest, the hind feet showed a drag gait, the forefoot stride length was significantly shortened and the stride width was significantly increased. Moreover, the gait of the hind foot and the stride length and width of the forefoot showed the most obvious improvement in the Cur@EXs ${ }^{-\mathrm{cl}-\mathrm{NGF}}$ group (Figure.6F). In addition, the BMS scores at multiple time points after injury in each group of mice further confirmed that the Cur@EXs ${ }^{-\mathrm{Cl}^{-N G F}}$ group had an excellent therapeutic effect on spinal cord injury (Figure. 6G).

\section{The role of Cur@EXs ${ }^{-\mathrm{cl}-\mathrm{NGF}}$ in the early stages of injury.}


We further explored the internal mechanism of Cur@EXs ${ }^{-\mathrm{cl}-\mathrm{NGF}}$ treatment for SCI. Spinal cord tissues from each group were removed $7 \mathrm{~d}$ after injury to observe the pathological changes. The spinal cord 5 $\mathrm{mm}$ above and below the injury point was collected to make a single cell suspension. The flow cytometry results showed a changed polarization state of monocytes/macrophages in spinal cord tissue. The proportion of $\mathrm{M} 1$ monocytes/macrophages ( $\mathrm{F} 4 / 80+, \mathrm{CD} 86+)$ was significantly higher than that of $\mathrm{M} 2$ monocytes/macrophages $(\mathrm{F} 4 / 80+, \mathrm{CD} 206+)$ in the NS group. Among them, the proportion of M1 monocytes/macrophages in total monocytes/macrophages increased to approximately $85.43 \%$, while the proportion of M2 monocytes/macrophages was only $5 \%$. With different treatments, the polarization state of monocytes/macrophages changed accordingly. Cur@EXs ${ }^{- \text {cl-NGF }}$ displayed the most obvious regulatory effect on monocyte/macrophage polarization, balancing the ratio of proinflammatory M1 monocytes/macrophages and anti-inflammatory M2 monocytes/macrophages (Figure. 7A, Figure. 7B). Subsequently, we detected the expression of proinflammatory factors (TNF-a, IL-1 $\beta$, and IL-6) and antiinflammatory factors (TGF- $\beta$ ) in the injured spinal cord tissue. The results indicated that compared with the NS treatment group, proinflammatory factors in the spinal cord tissue of the Cur@EXs ${ }^{-{ }^{-N}}{ }^{-N F}$ group were significantly reduced, while anti-inflammatory factors were significantly increased (Figure. 7D). Moreover, we observed the neuron survival status and apoptosis at the injury point by immunofluorescence staining of the surviving neuronal cells (labeled with anti-NurN antibody) and apoptotic protein c-caspase3 (labeled with anti- c-caspase3 antibody) in each group of mice (Figure. 7C). The NurN fluorescence intensity statistical analysis showed that following treatment of EXs ${ }^{-\mathrm{Cl}-\mathrm{NGF}}$ and Cur@EXs ${ }^{-\mathrm{Cl}-\mathrm{NGF}}$, the number of surviving neurons increased significantly, and the number of surviving neurons was greatest in the Cur@EXs ${ }^{-{ }^{-N}-N G F}$ group (Figure. 7E). The fluorescence intensity statistical analysis of Caspase-3 showed that after Cur@EXs ${ }^{-\mathrm{cl}-\mathrm{NGF}}$ treatment, apoptosis of the lesion was

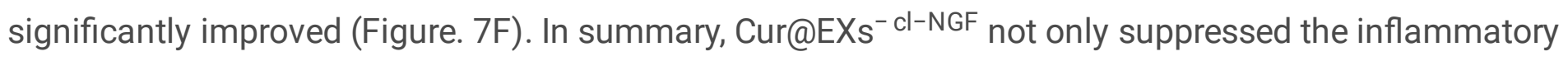
response by balancing the polarization ratio of macrophages but also improved the survival rate of damaged nerve cells, thereby improving the microenvironment of the injured site and promoting functional recovery after injury.

\section{Discussion}

An anti-inflammatory combined neuroprotection strategy is one of the most promising options for the treatment of $\mathrm{SCl}[8,32]$. Activation of microglia is one of the earliest inflammatory reactions after injury, and this activation is usually excessive [33]. Many proinflammatory factors are released into the damaged microenvironment, and many mononuclear macrophages are recruited to the inflammation site to form an uncontrollable inflammatory response [34]. We found that the microglia/macrophages that accumulated at the injury site in the early stage of injury were mainly the M1 type (Figure. S1), which indicated that the polarization of microglia/macrophages was severely imbalanced under the action of proinflammatory factors [35]. Therefore, regulating the polarization direction of microglia/macrophages to achieve the balance between M1 microglia/macrophages and M2 microglia/macrophages is a potential treatment strategy for uncontrollable inflammatory responses in $\mathrm{SCl}$ [36]. Previous studies have shown that EXs can change the polarization state of M1-type macrophages after being taken up by M1- 
type macrophages $[15,37]$. We further found that the ratio of monocytes/macrophages in peripheral blood leukocytes increased significantly within $7 \mathrm{~d}$ after injury and reached a peak on day 3 (Figure. S2). This finding implies that due to the recruitment of inflammation sites, EXs can actively target inflammation sites and exert inflammatory inhibitory effects [2,38].

In this study, the first step was to design a stable drug delivery vehicle with anti-inflammatory, neuroprotective and targeting capabilities. The results of western blotting and cell proliferation experiments showed that the modification process did not affect the activity of NGF or the function of EXs (Figure. 2l, Figure. 2K). Thus, the anti-inflammatory and targeting functions of EXs will not change with the addition of NGF. In vivo imaging results of the injured site showed that compared with RVs with only passive targeting capabilities, $\mathrm{EXs}^{-\mathrm{Cl}-\mathrm{NGF}}$ had a natural tendency to migrate to the injury site (Figure. $3 \mathrm{~A})$. The results of fluorescence imaging of the spinal cord and vital organs showed that in addition to the different accumulations in the spinal cord, free NGF was significantly accumulated in the kidneys, while NGF in $\mathrm{RVs}^{-{ }^{-\mathrm{Cl}}-\mathrm{NGF}}$ or EXs ${ }^{-\mathrm{Cl}^{-N G F}}$ was heavily accumulated in the spleen, especially in the EXs ${ }^{-}$ cl-NGF group (Figure. $3 C$ ). More importantly, apart from its active targeting ability, EXs also significantly prolonged the circulation time in the body of NGF (Figure. 3E). These advantages of EXs ${ }^{-\mathrm{Cl}^{-N G F}}$ greatly increased the amount of NGF that accumulated at the injured site and significantly improved the bioavailability of NGF. Considering that the target cells of EXs are inflammatory cells, the timely dissociation of NGF from EXs after reaching the injured site is also very meaningful. It has been reported that after $\mathrm{SCl}$, the concentration of MMP9 at the injured site increases significantly, and we also discovered this phenomenon through ELISA. CLSM and flow cytometry showed that NGF coupled with EXs through a cleavable linker could be effectively taken up by PC12 cells (Figure. 3G, Figure. 3H). Researchers who use exosomes as carriers for NGF often overlook the consumption of NGF by inflammatory cells such as microglia/macrophages. Therefore, it is necessary to use cleavable linkers to ensure that NGF reaches the injured site and can be internalized by nerve cells as much as possible instead of macrophages.

In vitro cell experiments confirmed that EXs reduced the expression of proinflammatory factors (TNF- $a$, IL$1 \beta$, IL-6) in the injured microenvironment and increased the secretion of anti-inflammatory factors (TGF- $\beta$ ) (Figure. 4C). Subsequently, we used flow cytometry to analyze the phenotype of the primary macrophages in the Transwell ${ }^{\mathrm{Tm}}$ coculture system. The results showed that under the action of EXs, the number of M1 macrophages was reduced by approximately $40 \%$, while the number of M2 macrophages was increased by approximately $10 \%$. The results suggested that although EXs significantly inhibited the inflammatory response, the macrophages were still in a proinflammatory polarized state. Thus, using EXs might not be sufficient to achieve a satisfactory therapeutic effect, so the drug carrier effect of EXs should also be fully utilized. The model drug Cur was loaded into EXs to improve the inflammationinhibiting effect of the system. As expected, compared with EXs ${ }^{-\mathrm{cl}-\mathrm{NGF}}$, Cur@EXs ${ }^{-\mathrm{cl}-\mathrm{NGF}}$ had a more obvious inhibitory effect on proinflammatory M1 macrophages and significantly promoted the secretion of anti-inflammatory factors and the expression of the macrophage marker protein M2 (ARG-1) (Figure. $5 F$, Figure. $5 \mathrm{G}$ ). The phenotypic verification results of microglia/macrophages accumulating at the injury 
site showed that Cur@EXs ${ }^{-\mathrm{Cl}-\mathrm{NGF}}$ rebalanced microglia/macrophage polarization (Figure.6A). This finding is of great significance for reducing the secondary damage caused by uncontrollable inflammation.

Moreover, NGF coupled with EXs by cleavable linkers could effectively inhibit the apoptosis of PC12 cells after injury. Flow cytometry and CLSM images suggested that although EXs could indirectly inhibit apoptosis of PC12 cells by reducing the inflammatory response [39, 40], the degree of inhibition was very limited (Figure. 4A, Figure. 4B). This result indicated that it was necessary to link NGF on the surface of EXs, which improved the survival rate of nerve cells. The results of in vivo experiments further validated this point. Because of the existence of NGF, Cur@EXs ${ }^{-\mathrm{Cl}-\mathrm{NGF}}$ greatly promoted the survival rate of neuronal cells in the early stage of injury, which increased the possibility of subsequent functional recovery (Figure. 6C). The results of motor function recovery also illustrated that Cur@EXs ${ }^{\text {cl-NGF }}$ had the best therapeutic effect, although Cur and EXs ${ }^{-\mathrm{Cl}-\mathrm{NGF}}$ also had different degrees of therapeutic effect (Figure. 7). Moreover, Cur@EXs ${ }^{-c^{-N G F}}$ not only had an excellent therapeutic effect but also presented superior safety (Figure. S4, Figure. S5). Combining all the experimental results, we believe that Cur@EXs' $\mathrm{cl}-\mathrm{NGF}$, with both inflammation targeting and inhibition and neuroprotection, can provide inspiration for the clinical treatment of SCl.

\section{Conclusion}

In this study, we designed multifunctional engineered exosomes (Cur@EXs ${ }^{-\mathrm{cl}-\mathrm{NGF}}$ ) to promote the recovery of motor function after $\mathrm{SCl}$. The combination of exosomes and curcumin effectively changed the polarization state of M1 macrophages after injury and limited the damage to spinal cord tissue caused by uncontrollable inflammation. Cur@EXs ${ }^{-\mathrm{Cl}-\mathrm{NGF}}$ also achieved the transfer of exogenous NGF to the injured site and improved the survival rate of injured nerve cells. Both in vivo and in vitro tests demonstrated that the present design could provide new treatment options for SCl.

\section{Materials And Methods}

\section{Exosome isolation}

Extraction and induction of peritoneal macrophages: The abdominal skin of the mice was disinfected with ethanol, and $1 \mathrm{~mL}$ of $5 \%$ starch broth solution was injected into the abdominal cavity of each mouse. After $48 \mathrm{~h}$, the mice were sacrificed by cervical dislocation. The peritoneum of the mice was exposed under aseptic conditions, and the peritoneal cavity was injected with $5 \mathrm{~mL}$ precooled Dulbecco's Modified Eagle's Medium (DMEM). After gently massaging the mouse's abdomen for 5 min, a 5-mL syringe was used to repeatedly flush the peritoneal cavity lavage fluid twice, and then the lavage fluid was recovered. The lavage fluid was centrifuged at $1000 \mathrm{rpm}$ for $10 \mathrm{~min}$, the supernatant was discarded, and the peritoneal cell concentration was adjusted to $1 \times 10^{6} / \mathrm{mL}$. One milliliter per well of the cell suspension was inoculated in a 12-well culture plate and placed in an incubator at $37^{\circ} \mathrm{C}$ with $5 \%$ carbon 
dioxide and saturated humidity to allow the macrophages to adhere to the wall. After incubating for $2 \mathrm{~h}$ to $4 \mathrm{~h}$, the culture supernatant was discarded, and the cells were cultured in DMEM supplemented with exosome-free $10 \%$ fetal bovine serum (FBS) and 1\% penicillin-streptomycin (PS). After $24 \mathrm{~h}$, interleukin-4 (IL-4) $(20 \mu \mathrm{g} / \mathrm{mL})$ was added to the medium to induce the differentiation of primary macrophages into M2 macrophages.

Exosome isolation: The cell supernatant of primary M2 macrophages was collected and placed in a centrifuge tube, which was sequentially centrifuged at $300 \mathrm{~g}$ for $10 \mathrm{~min}, 2,000 \mathrm{~g}$ for $10 \mathrm{~min}, 10,000 \mathrm{~g}$ for $30 \mathrm{~min}$, and $100,000 \mathrm{~g}$ for $70 \mathrm{~min}$ at $4^{\circ} \mathrm{C}$. The obtained precipitates were suspended in phosphatebuffered saline (PBS) and centrifuged at 100,000 g for 70 min to wash away impurities. The exosomes were resuspended in PBS for further characterization.

The preparation of EXS ${ }^{-\mathrm{Cl}-\mathrm{NGF}}$ and Cur@EXS ${ }^{-\mathrm{Cl}-\mathrm{NGF}}$.

Modification of NGF with cleavable peptide substrate (NHS-Arg-Val-Gly-Leu-Pro-(6-Mal), RVGLP-(6-Mal)): The peptide $(0.5 \mathrm{mg} / \mathrm{mL})$ was added to the NGF solution $(1 \mathrm{mg} / \mathrm{mL})$ at a molar ratio of $50: 1$, keeping the linker in excess. The mixed solution was incubated at $4^{\circ} \mathrm{C}$ in PBS (pH 7.4) overnight. Then, the samples were filtered using a centrifugal filter device (Amicon Ultra-0.5, Millipore Co, Germany) at 7,000 g for 30 min to remove the excess linker. Next, the NGF was linked to EXs. The exosomes were pretreated with 1 $\mathrm{mM} \mathrm{TCEP}$ at $37^{\circ} \mathrm{C}$ for 30 min to break the disulfide bonds on the surface of the exosomes and expose the sulfhydryl groups. Then, "-cl-NGF" was dissolved in PBS (pH 7.4) and reacted with the EXs at $25^{\circ} \mathrm{C}$ for $1 \mathrm{~h}$. Finally, the EXS ${ }^{-\mathrm{Cl}-N G F}$ was washed and collected by dialysis. Cur was dissolved in a 1:1 mixed solution of ethanol and acetonitrile. PBS was added to the mixed solution to a concentration of the organic solvent of $10 \%$. EXs ${ }^{- \text {cl-NGF }}$ was added at a concentration of $20 \%$ curcumin to the solution. Cur@EXs cl-NGF was prepared by ultrasonic soaking using a $40-\mathrm{kHz}$ and $100-\mathrm{W}$ intermittent ultrasonic cleaner for $15 \mathrm{~min}$ and washing by centrifugation at $5000 \mathrm{rpm}$ three times with a $100-\mathrm{kDa}$ ultrafiltration tube. The loading capacity or percent drug load was calculated using the following formula.

Percent drug load $=\left(\right.$ amount of Cur in Cur@EXS ${ }^{-\mathrm{cl}-\mathrm{NGF}} /$ amount of Cur@EXS $\left.{ }^{-\mathrm{cl}-\mathrm{NGF}}\right){ }^{\star} 100$ Characterization of EXS ${ }^{-\mathrm{Cl}-\mathrm{NGF}}$ and Cur@EXS ${ }^{-\mathrm{Cl}-\mathrm{NGF}}$

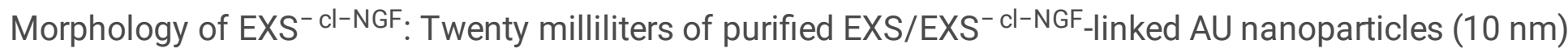
were added onto a carbon-coated copper grid for $20 \mathrm{~min}$, stained with $2 \%$ uranyl acetate for $1 \mathrm{~min}$, and then washed with distilled water. Transmission electron microscopy (TEM) images were obtained on a JEM-1400 microscope (JEOL Japan) with an accelerating voltage of $120 \mathrm{kV}$. The size distribution and zeta potential of EXS/EXS ${ }^{-}$cl-NGF were analyzed on a Zetaview (Partical Metrix Germany).

Western blot analysis: The expression of special marker proteins of EXS/EXS ${ }^{-\mathrm{Cl}-\mathrm{NGF}}$ was detected using a western blot analyzer with BeyoECL Plus (Beyotime, Beijing, China). The primary antibodies used were as follows: rabbit anti-CD9 (1:1000, Abcam, Cambridge, UK), rabbit anti-TSG101 (1:1000, Abcam, 
Cambridge, UK), rabbit anti-CD206 (1:1000, Cell Signaling Technology, MA, USA), rabbit anti-IL10 (1:1000, Cell Signaling Technology, MA, USA), rabbit anti-CCR2 (1:1000, Cell Signaling Technology, MA, USA), rabbit anti-ARG-1 (1:1000, Cell Signaling Technology, MA, USA), rabbit anti-NGF (1:1000, Cell Signaling Technology, MA, USA), and mouse anti- $\beta$-actin (1:1000, Abcam, Cambridge, UK).

Colocalization analysis of EXS ${ }^{-\mathrm{cl}-\mathrm{NGF}}$ and Cur@EXS ${ }^{-\mathrm{cl}-\mathrm{NGF}}$ : First, EXs were dyed with DID, and NGF was labeled with CY3. Subsequently, the double fluorescently labeled nanomaterial EXS ${ }^{-\mathrm{Cl}-\mathrm{NGF}}$ was prepared using the above method. EXS ${ }^{-\mathrm{Cl}-N G F}$ was imaged by confocal laser scanning microscopy (CLSM) (Nikon A1 Japan) and visualized with stimulated emission depletion (STED) (Leica SP8, Germany).

Release and biological activity of NGF from EXS ${ }^{-\mathrm{Cl}-\mathrm{NGF}}$ : MMP-9 was added to a PBS solution of EXS $\mathrm{Cl}-\mathrm{NGF}$ with/without inhibitor, and the supernatant was removed at different time points. After ultrafiltration, the content of NGF in the filtrate was detected with an NGF ELISA kit. The biological activity of NGF was detected by the MTT test in PC12 cells, in which different concentrations of NGF from EXS ${ }^{-}$ $\mathrm{cl}-\mathrm{NGF} /$ free NGF were added to the culture medium of PC12 cells.

Construction of the Transwell ${ }^{\mathrm{TM}}$ coculture model

The Transwell ${ }^{\text {TM }}$ coculture model was built with Transwell ${ }^{\text {TM }}$ plates (pore size of $8 \mu \mathrm{m}$, Corning, USA), and $0.5-1 \times 10^{5}$ PC1 2 cells were loaded in each upper chamber. Primary macrophages were removed from the mouse abdominal cavity and pretreated in the lower chamber with lipopolysaccharide (LPS) $(0.1 \mu \mathrm{g} / \mathrm{mL})$ for $24 \mathrm{~h}$ before building the Transwell ${ }^{\mathrm{TM}}$ coculture model. All the cells were cultured in DMEM (Gibco, Grand Island, NY, USA) supplemented with fetal bovine serum (10\%), penicillin (100 units $/ \mathrm{mL}$ ), and streptomycin $(100 \mu \mathrm{g} / \mathrm{mL})$ (Gibco, Grand Island, NY, USA) at $37^{\circ} \mathrm{C}$ in a humidified atmosphere containing $\mathrm{CO}_{2}(5 \%)$.

Apoptosis test

For the apoptosis experiment of PC12 cells, PC12 cells were loaded in each upper chamber and stimulated with $200 \mu \mathrm{M}$ hydrogen peroxide $\left(\mathrm{H}_{2} \mathrm{O}_{2}\right)$ for $12 \mathrm{~h}$ to simulate damage to cells caused by oxidative stress. After changing to normal medium, primary M1 macrophages were placed in the lower layer of the Transwell ${ }^{\text {TM }}$ coculture model for $12 \mathrm{~h}$. Different nanoparticles (PBS, EXS, NGF, EXS ${ }^{-N G F}$, EXS ${ }^{-}$ cl-NGF) were added to the different chambers, and after $12 \mathrm{~h}$, the apoptosis rate of PC12 cells and the macrophage polarization level in each chamber were evaluated by flow cytometry and CLSM.

Flow Cytometry

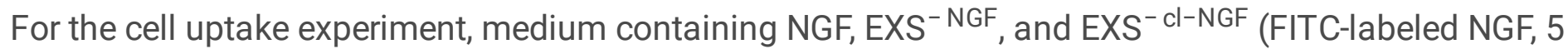
$\mu \mathrm{g} / \mathrm{mL}$ ) was added to the Transwell ${ }^{\mathrm{TM}}$ coculture model. At the different time points, cells were removed and resuspended in standing buffer to assess the fluorescence of different cells in different groups (Beckman Coulter, USA). 
For the apoptosis rate of PC12 cells, the Transwel ${ }^{\text {TM }}$ coculture model was removed after different stimulations, and the upper PC12 cells were resuspended. A total of $10^{5} \mathrm{PC} 12$ cells were mixed in $100 \mu \mathrm{L}$ of binding buffer. Cells were stained with Annexin V-Alexa Fluor 488/PI for $15 \mathrm{~min}$ at room temperature to assess cell apoptosis.

For macrophage polarization, macrophage cells were removed, and flow cytometry antibodies were added for 30 min at $4^{\circ} \mathrm{C}$. M1 macrophages were labeled with F4/80 (Biolegend, USA) and CD86 (Biolegend, USA), and M2 macrophages were labeled with F4/80 (Biolegend, USA) and CD206 (Biolegend, USA) to evaluate the differentiation of macrophage phenotypes.

Animals and the SCl model

Adult male C56BL/ 6 mice (8-10 weeks old, 22-25 g) were used. All animal experiments were approved by the Animal Protection and Use Committee of Jinzhou Medical University. A contusion-induced SCI model was developed by an improved weightlessness method. In brief, after intraperitoneal anesthesia with $1 \%$ sodium pentobarbital $(50 \mathrm{mg} / \mathrm{kg}$ ), the mice were fixed on a sterile operating table, fully exposing the T9 spinal cord. Using a 12.5-g impactor device (diameter: $2 \mathrm{~mm}$ ), the uniform height was $5 \mathrm{~cm}$ down to the spinal cord, resulting in a moderate spinal cord contusion. After successful modeling, the surgical incision was sutured layer by layer, and antibiotics were given to prevent infection. From $2 \mathrm{~d}$ after surgery, the mouse bladder was massaged twice a day to help them urinate until the mice could urinate spontaneously. Sham-operated mice underwent the same procedures, except for contusion of the spinal cord. To investigate the treatment effect in vivo, $100 \mu \mathrm{L}$ of $2 \mathrm{mg} / \mathrm{mL}$ Cur, EXS ${ }^{-\mathrm{Cl}-\mathrm{NGF}}$ or Cur@EXs ${ }^{-\mathrm{cl}^{-N G F}}$ was injected through the tail vein at $2 \mathrm{~h}, 2 \mathrm{~d}, 4 \mathrm{~d}$, and $6 \mathrm{~d}$ after injury.

In vivo imaging

For in vivo imaging of nanoparticles, $100 \mu \mathrm{L}$ of NGF, red vesicles (RVs $\left.{ }^{-\mathrm{Cl}-\mathrm{NGF}}\right)$, or EXs ${ }^{-\mathrm{Cl}-\mathrm{NGF}}(2 \mathrm{mg} / \mathrm{mL}$, Alexa Fluor ${ }^{\circledR}$ 647-labeled NGF) was injected via the tail vein. At different time points, mice were imaged using a Kodak Imaging System FX Pro to evaluate the fluorescence signal distribution in vivo. Twelve hours after tail vein injection, the mice were sacrificed by cervical dislocation, and the mouse heart, liver, spleen, lung, kidney, and spinal cord were removed for fluorescence imaging.

Immunofluorescence staining

For the cell uptake experiment, medium containing NGF, EXS ${ }^{-N G F}$, and EXS ${ }^{-\mathrm{Cl}-\mathrm{NGF}}$ (FITC-labeled NGF, 5 $\mu \mathrm{g} / \mathrm{mL}$ ) was added to the Transwell ${ }^{\mathrm{Tm}}$ coculture model for $6 \mathrm{~h}$. Cells were removed from the incubator, and the cells were fixed with immune tissue fixative. Finally, the cell membrane was labeled with phalloidin 561, and the cell nucleus was labeled with 4',6-diamidino-2-phenylindole (DAPI).

For immunofluorescent dual-labeling staining, cells or frozen sections were fixed with immune tissue fixative and incubated with Triton X-100 (0.3\%) followed by goat serum (10\%) for $2 \mathrm{~h}$. The cells were incubated with the first antibody overnight at $4^{\circ} \mathrm{C}$, washed, and then incubated with Alexa Fluor 488 goat 
anti-rabbit IgG or Alexa Fluor 594 goat anti-mouse IgG (A-11034/A-11005, 1:250, Thermo Fisher Science) for $2 \mathrm{~h}$ at room temperature. Next, the cell nucleus was stained with DAPI. The primary antibodies were as follows: rabbit anti-F4/80 (1:200, Abcam, Cambridge, UK), rabbit anti- cleaved-Caspase3 (c-caspase3) (1:1000, Abcam, Cambridge, UK), mouse anti-ARG-1 (1:1000, Cell Signaling Technology, MA, USA), rabbit anti-iNOS (1:1000, Cell Signaling Technology, MA, USA), rabbit anti-NeuN (1:50, Cell Signaling Technology, MA, USA), rabbit anti-GFAP (1:200, Cell Signaling Technology, MA, USA), and rabbit anti- $\beta 3-$ Tubulin ( $1: 200$, Cell Signaling Technology, MA, USA).

Behavioral analysis

The basso mouse scale (BMS) exercise rating scale was used to evaluate the functional recovery of injured animals on days $1,3,5,7,14,21$ and 28 according to scores ranging from 0 to 9 (9 for complete normality and 0 for complete paralysis). The animals were placed in the open field and observed for 4 min. Three examiners who did not know the mouse grouping observed and scored the mouse's ankle joints, the touch degree of the sole and dorsum of the foot, trunk stability and tail position. The experiment was repeated three times.

\section{Statistical analysis}

All data are expressed as the average value of the data distribution evaluated by the Shapiro-Wilk test. The Mann-Whitney $U$ test was used for two-group comparisons. One-way ANOVA and Bonferroni post hoc tests were used to compare more than two groups. All data were graphed and statistically analyzed using

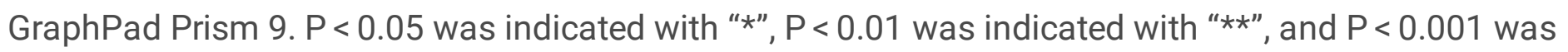



\section{Abbreviations}

SCl: spinal cord injury; NGF: nerve growth factor; Cur: curcumin; IL-10: interleukin-10; TGF- $\beta$ : transforming growth factor; TNF-a: tumor necrosis factor-a; IL-1 $\beta$ : interleukin-1 $\beta$; IL-6: interleukin-6; CCR2: chemokine receptor type 2; MMP9: matrix metalloproteinase 9; IL-4: interleukin-4; TEM: transmission electron microscopy; CLSM: confocal laser scanning microscopy; STED: stimulated emission depletion; BMS: basso mouse scale

\section{Declarations}

\section{Acknowledgements}

We gratefully thank all colleagues in the Liaoning Provincial Key Laboratory of Brain and Spinal Cord Injury. This work was supported by grants from the National Natural Science Foundation of China (no. 81671907, 81871556, 82072165) and Liaoning Revitalization Talents Program (no. XLYC1902108) and Scientific Research Project of the Educational Department of Liaoning Province (no. JYTQN201917). 


\section{Authors' contributions}

$\mathrm{XM}$ and $\mathrm{CW}$ conceived and designed the experiments. $\mathrm{CZ}$ executed the experiments, analyzed the data, and wrote the manuscript. DL and ZW performed the in vivo experiments. $\mathrm{HH}, \mathrm{JA}, \mathrm{ZG}$ and $\mathrm{KZ}$ edited the manuscript. XM and HT supervised the project and made final approval of manuscript. All authors read and approved the fnal manuscript.

\section{Funding}

This work was supported by grants from the National Natural Science Foundation of China (no. 81671907, 81871556, 82072165) and Liaoning Revitalization Talents Program (no. XLYC1902108) and Scientific Research Project of the Educational Department of Liaoning Province (no. JYTQN201917).

\section{Availability of data and materials}

The datasets used and/or analysed during the current study are available from the corresponding author on reasonable request.

\section{Ethics approval and consent to participate}

All animal experiments were approved by the Animal Protection and Use Committee of Jinzhou Medical University.

\section{Consent for publication}

Not applicable.

\section{Competing interests}

The authors declare that they have no known competing financial interests or personal relationships that could have appeared to influence the work reported in this paper.

\section{Author details}

${ }^{1}$ Department of Orthopedics, The First Affifiliated Hospital of Jinzhou Medical University, No. 2, Section 5, Renmin Street Jinzhou 121002, Liaoning, China. ${ }^{2}$ Key Laboratory of Brain and Spinal Cord Injury of Liaoning Province, No. 40, Songpo Road, Jinzhou 121002, Liaoning, China. ${ }^{3}$ Pharmacy School, Jinzhou Medical University, No. 40, Songpo Road, Jinzhou 121002, Liaoning, China. ${ }^{4}$ Department of Histology and Embryology, Jinzhou Medical University, No. 40, Songpo Road, Jinzhou 121002, Liaoning, China

\section{References}

1. Geisler FH, Coleman WP, Benzel E, Ducker T, Hurlbert RJ. Spinal cord injury. Lancet. 2002;360:18834. 
2. Nogueira PC, Rabeh SA, Caliri MH, Dantas RA, Haas VJ. Burden of care and its impact on healthrelated quality of life of caregivers of individuals with spinal cord injury. Rev Lat Am Enfermagem. 2012;20:1048-56.

3. David G, Mohammadi S, Martin AR, Cohen-Adad J, Weiskopf N, Thompson A, et al. Traumatic and nontraumatic spinal cord injury: pathological insights from neuroimaging. Nat Rev Neurol. 2019;15:718-31.

4. Ahuja CS, Wilson JR, Nori S, Kotter Mark RN, Druschel C, Curt A, et al. Traumatic spinal cord injury. Nat Rev Dis Primers. 2017;3:17018.

5. Ahuja CS, Mothe A, Khazaei M, Badhiwala JH, Gilbert EA, van der Kooy D, et al. Fehlings, The leading edge: Emerging neuroprotective and neuroregenerative cell-based therapies for spinal cord injury. Stem Cells Transl Med. 2020;9:1509-30.

6. Ren H, Chen X, Tian M, Zhou J, Ouyang H, Zhang Z. Regulation of Inflammatory Cytokines for Spinal Cord Injury Repair Through Local Delivery of Therapeutic Agents. Adv Sci. 2018;5:1800529.

7. Beattie MS. Inflammation and apoptosis: linked therapeutic targets in spinal cord injury. Trends Mol Med. 2004;10:580-3.

8. Song YH, Agrawal NK, Griffin JM, Schmidt CE. Recent advances in nanotherapeutic strategies for spinal cord injury repair. Adv Drug Deliv Rev. 2019;148:38-59.

9. Yong T, Zhang X, Bie N, Zhang H, Zhang X, Li F, et al. Tumor exosome-based nanoparticles are efficient drug carriers for chemotherapy. Nat Commun. 2019;10:3838.

10. Liao W, Du Y, Zhang C, Pan F, Yao Y, Zhang T, et al. Exosomes: The next generation of endogenous nanomaterials for advanced drug delivery and therapy. Acta Biomater. 2019;86:1-14.

11. Kim HY, Kumar H, Jo MJ, Kim J, Yoon JK, Lee JR, et al. Therapeutic Efficacy-Potentiated and Diseased Organ-Targeting Nanovesicles Derived from Mesenchymal Stem Cells for Spinal Cord Injury Treatment. Nano Lett. 2018;18:4965-75.

12. David S, Kroner A. Repertoire of microglial and macrophage responses after spinal cord injury. Nat Rev Neurosci. 2011;12:388-99.

13. Wu G, Zhang J, Zhao Q, Zhuang W, Ding J, Zhang C, et al. Molecularly Engineered MacrophageDerived Exosomes with Inflammation Tropism and Intrinsic Heme Biosynthesis for Atherosclerosis Treatment. Angew Chem Int Ed Engl. 2020;59:4068-74.

14. Song Y, Li Z, He T, Qu M, Jiang L, Li W, et al. M2 microglia-derived exosomes protect the mouse brain from ischemia-reperfusion injury via exosomal miR-124. Theranostics. 2019;9:2910-23.

15. Gao ZS, Zhang CJ, Xia N, Tian H, Li DY, Lin JQ, et al. Berberine-loaded M2 macrophage-derived exosomes for spinal cord injury therapy. Acta Biomater. 2021;126:211-23.

16. Convertino D, Fabbri F, Mishra N, Mainardi M, Cappello V, Testa G, et al. Graphene Promotes Axon Elongation through Local Stall of Nerve Growth Factor Signaling Endosomes. Nano Lett. 2020;20:3633-41. 
17. Xu D, Wu D, Qin M, Nih LR, Liu C, Cao Z, et al. Efficient Delivery of Nerve Growth Factors to the Central Nervous System for Neural Regeneration. Adv Mater. 2019;31:e1900727.

18. Yang J, Wu S, Hou L, Zhu D, Yin S, Yang G, Wang Y. Therapeutic Effects of Simultaneous Delivery of Nerve Growth Factor mRNA and Protein via Exosomes on Cerebral Ischemia. Mol Ther Nucleic Acids. 2020;21:512-22.

19. Ernfors $P$, Henschen A, Olson L, Persson H. Expression of nerve growth factor receptor mRNA is developmentally regulated and increased after axotomy in rat spinal cord motoneurons. Neuron. 1989;2:1605-13.

20. Tria MA, Fusco M, Vantini G, Mariot R. Pharmacokinetics of nerve growth factor (NGF) following different routes of administration to adult rats. Exp Neurol. 1994;127:178-83.

21. Yang Q, Peng J, Shi K, Xiao Y, Liu Q, Han R, et al. Rationally designed peptide-conjugated gold/platinum nanosystem with active tumor-targeting for enhancing tumor photothermalimmunotherapy. J Control Release. 2019;308:29-43.

22. Holz FG, Schmitz-Valckenberg S, Fleckenstein M. Recent developments in the treatment of agerelated macular degeneration. J Clin Invest. 2014;124:1430-8.

23. Miranpuri GS, Nguyen J, Moreno N, Yutuc NA, Kim J, Buttar S, et al. Folic Acid Modulates Matrix Metalloproteinase-9 Expression Following Spinal Cord Injury. Ann Neurosci. 2019;26:60-5.

24. Kawasaki Y, Xu ZZ, Wang X, Park JY, Zhuang ZY, Tan PH, et al. Distinct roles of matrix metalloproteases in the early- and late-phase development of neuropathic pain. Nat Med. 2008;14:331-6.

25. Boyd JG, Gordon T. Neurotrophic factors and their receptors in axonal regeneration and functional recovery after peripheral nerve injury. Mol Neurobiol. 2003;27:277-324.

26. Liu L, Li Y, Peng H, Liu R, Ji W, Shi Z, et al. Targeted exosome coating gene-chem nanocomplex as "nanoscavenger" for clearing a-synuclein and immune activation of Parkinson's disease. Sci Adv. 2020;6:eaba3967.

27. Dou G, Tian R, Liu X, Yuan P, Ye Q, Liu J, et al. Chimeric apoptotic bodies functionalized with natural membrane and modular delivery system for inflammation modulation. Sci Adv. 2020;6:eaba2987.

28. Mahjoob M, Stochaj U. Curcumin nanoformulations to combat aging-related diseases. Ageing Res Rev. 2021;69:101364.

29. Lu L, Qi S, Chen Y, Luo H, Huang S, Yu X, et al. Targeted immunomodulation of inflammatory monocytes across the blood-brain barrier by curcumin-loaded nanoparticles delays the progression of experimental autoimmune encephalomyelitis. Biomaterials. 2020;245:119987.

30. Geremia NM, Bao F, Rosenzweig TE, Hryciw T, Weaver L, Dekaban GA, et al. CD11d Antibody Treatment Improves Recovery in Spinal Cord-Injured Mice. J Neurotrauma. 2012;29:539-50.

31. Guo S, Perets N, Betzer O, Ben-Shaul S, Sheinin A, Michaelevski I, et al. Intranasal Delivery of Mesenchymal Stem Cell Derived Exosomes Loaded with Phosphatase and Tensin Homolog siRNA Repairs Complete Spinal Cord Injury. ACS Nano. 2019;13:10015-28. 
32. He Z, Zang H, Zhu L, Huang K, Yi T, Zhang S, et al. An anti-inflammatory peptide and brain-derived neurotrophic factor-modified hyaluronan-methylcellulose hydrogel promotes nerve regeneration in rats with spinal cord injury. Int J Nanomedicine. 2019;14:721-32.

33. Bellver-Landete $\mathrm{V}$, Bretheau $\mathrm{F}$, Mailhot $\mathrm{B}$, Vallières $\mathrm{N}$, Lessard $\mathrm{M}$, Janelle $\mathrm{ME}$, et al. Microglia are an essential component of the neuroprotective scar that forms after spinal cord injury. Nat Commun. 2019;10:518.

34. Yong YFH, Rawji Khalil S, Ghorbani S, Xue M, Yong VM. The benefits of neuroinflammation for the repair of the injured central nervous system. Cell Mol Immunol. 2019;16:540-6.

35. Thawer SG, Mawhinney L, Chadwick K, Chickera SN, Weaver LC, Brown A, et al. Temporal changes in monocyte and macrophage subsets and microglial macrophages following spinal cord injury in the Lys-Egfp-ki mouse model. J Neuroimmunol. 2013;261:7-20.

36. Papa S, Caron I, Erba E, Panini N, De PM, Mariani A, et al. Early modulation of pro-inflammatory microglia by minocycline loaded nanoparticles confers long lasting protection after spinal cord injury. Biomaterials. 2016;75:13-24.

37. Xia Y, Rao L, Yao H, Wang Z, Ning P, Chen X. Engineering Macrophages for Cancer Immunotherapy and Drug Delivery. Adv Mater. 2020;32:e2002054.

38. Pineau I, Lacroix S. Proinflammatory cytokine synthesis in the injured mouse spinal cord: multiphasic expression pattern and identification of the cell types involved. J Comp Neurol. 2007;500:267-85.

39. Karova K, Wainwright JV, Machova-Urdzikova L, Pisal RV, Schmidt M, Jendelova P, et al.

Transplantation of neural precursors generated from spinal progenitor cells reduces inflammation in spinal cord injury via NF-KB pathway inhibition. J Neuroinflammation. 2019;16:12.

40. Zhang S, Chuah SJ, Lai RC, Hui JHP, Lim SK, Toh WS. MSC exosomes mediate cartilage repair by enhancing proliferation, attenuating apoptosis and modulating immune reactivity. Biomaterials. 2018;156:16-27.

\section{Figures}




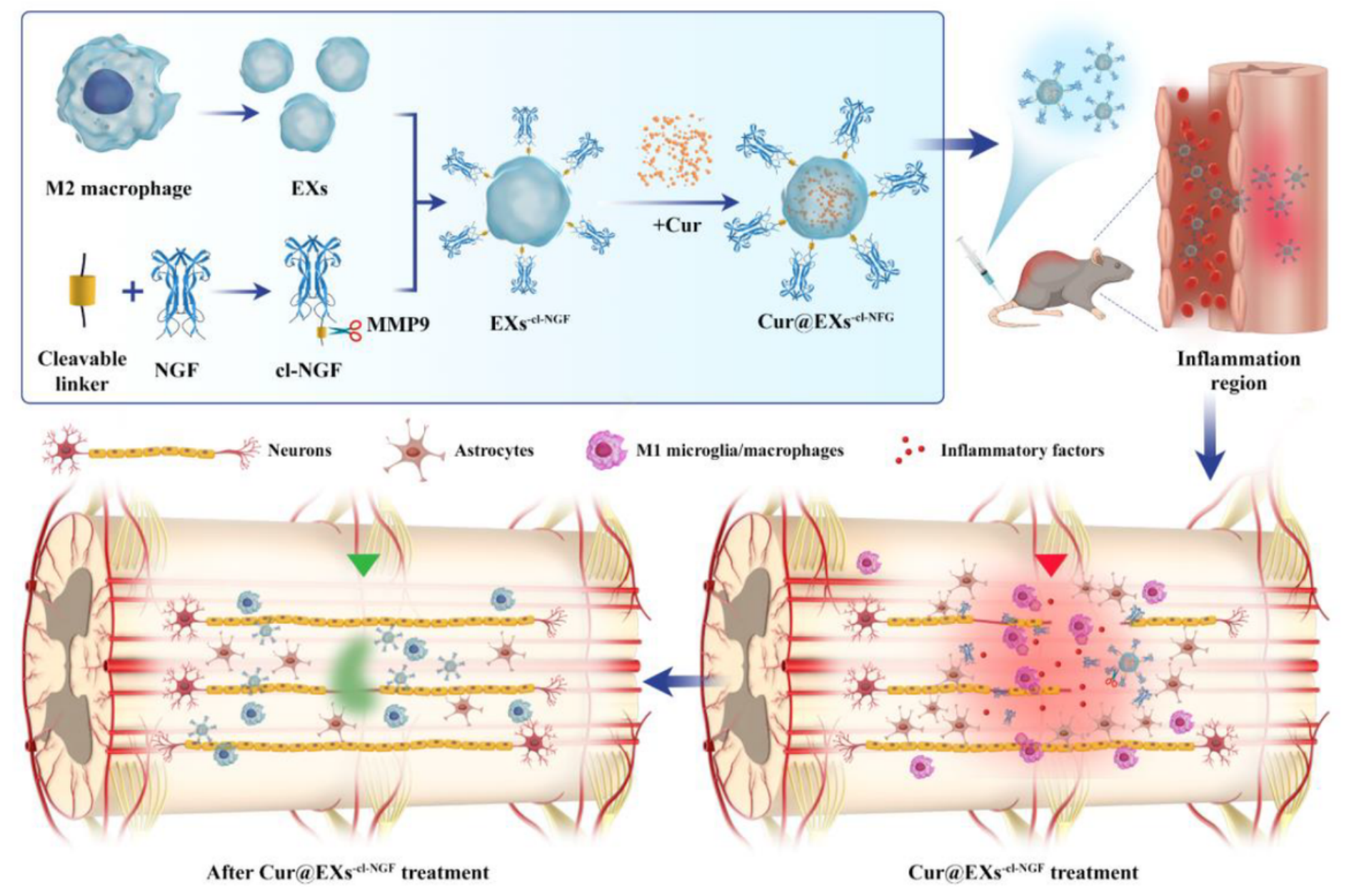

Figure 1

Scheme of engineered exosomes derived from primary M2 macrophages preparation and spinal cord injury therapy. 
A

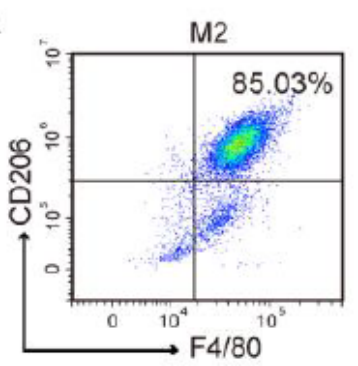

F

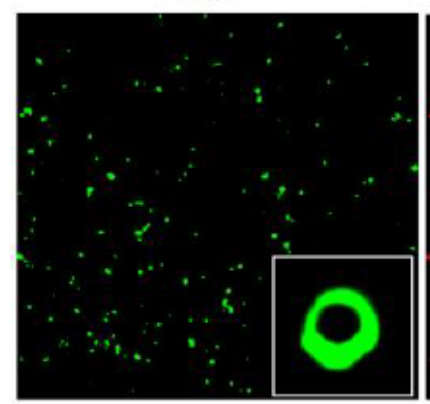

B $\mathrm{M} \odot \mathrm{M} \quad \mathrm{C}$

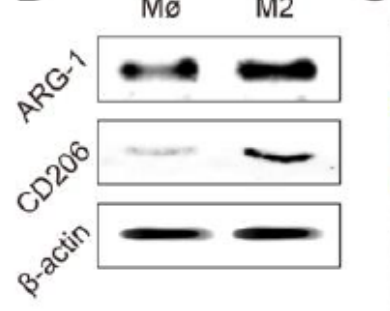

D

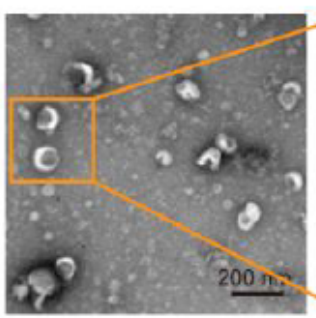

Merge

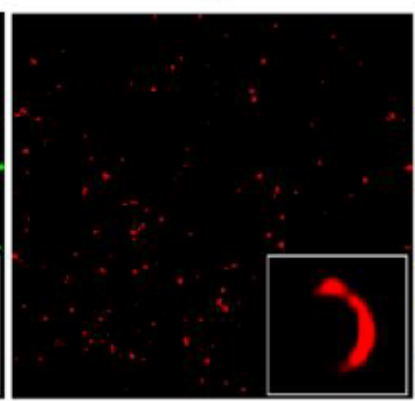

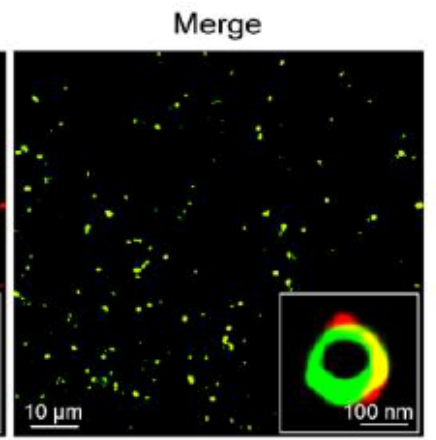

G

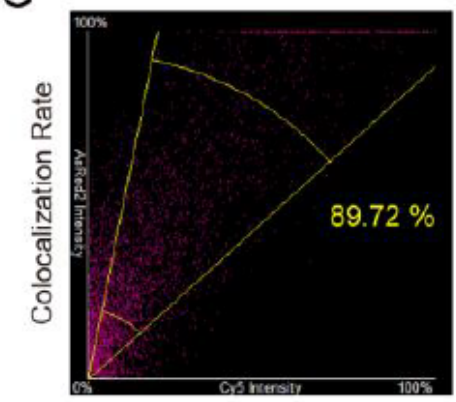

$\mathrm{H}$

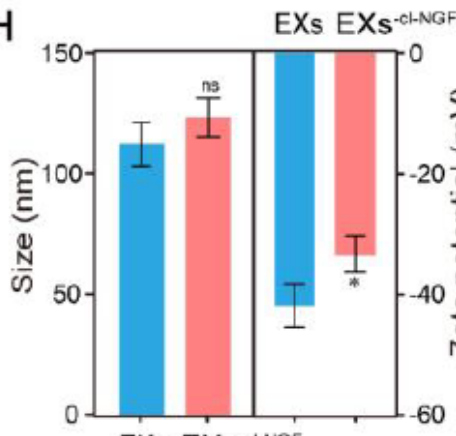

EXs EXs-el-NGF

L

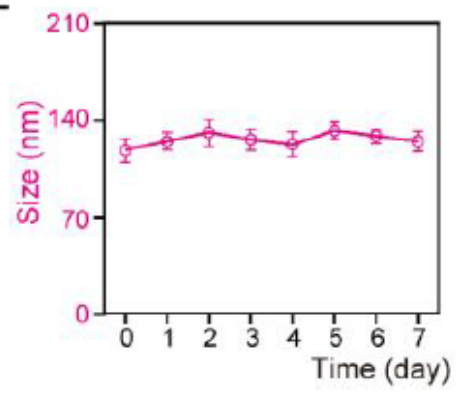

$\mathrm{J}$

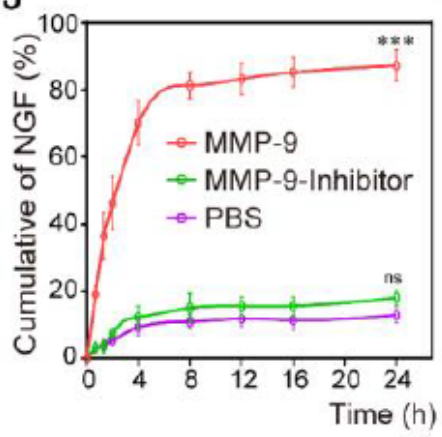

N Before lyophilization

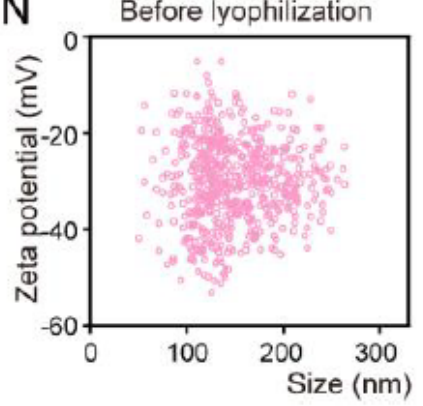

E

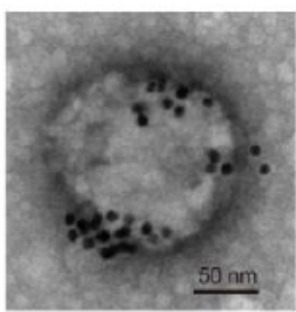

$\mathrm{K}$

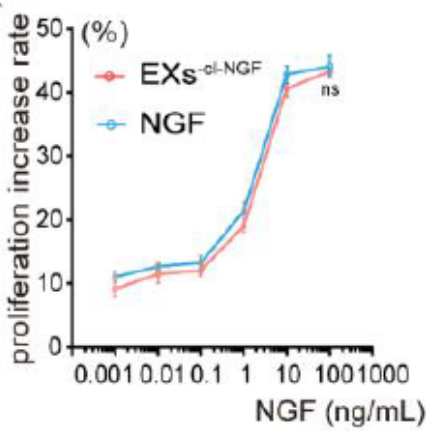

0

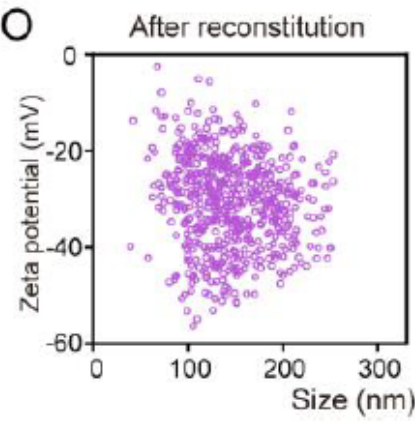

\section{Figure 2}

Preparation and characterization of EXs-cl-NGF. (A) Flow cytometry analysis of primary macrophages at $48 \mathrm{~h}$ after treatments with IL-4. (Numbers indicated percent F4/80+, CD206+ cells). (B) Western blotting analysis of primary Mø and M2 macrophages. ARG-1 and CD206 was a marker of M2 macrophages. (C, D) TEM imaging of EXs. (E) TEM images of EXs-Cl-NGF binded with secondary immune-antibody-linked Au nanoparticles. (F) CLSM images and the STED images (enlarged illustration in lower right corner) of EXs-cl-NGF, EXs (green) and NGF (red). (G) The colocalization rate of EXs and NGF. (H) The size and zeta potentials of EXs before and after loading NGF. (I) Western blotting analysis of EXs and EXs-cl-NGF. CD9 and TSG101 was a marker of exosomes, IL-10 was a functional protein of M2 macrophages and CCR2 
was Inflammatory chemotactic protein. $(\mathrm{J})$ The release rate of NGF under different conditions (PBS, MMP9 + inhibitor or MMP9). (K) Comparison of the activity of NGF released from EXs-cl-NGF to the original NGF. (L, M) The stability of particle size and zeta potentials of EXs-cl-NGF in PBS for 7 days. (N, O) Before lyophilization and after reconstitution, the particle size and Zeta potential of EXs-cl-NGF. Data presented the mean $\pm S D\left(n=3\right.$ per group) $\left({ }^{*} P<0.05\right.$, ${ }^{* \star *} P<0.001$ and $n s$ : not significant)
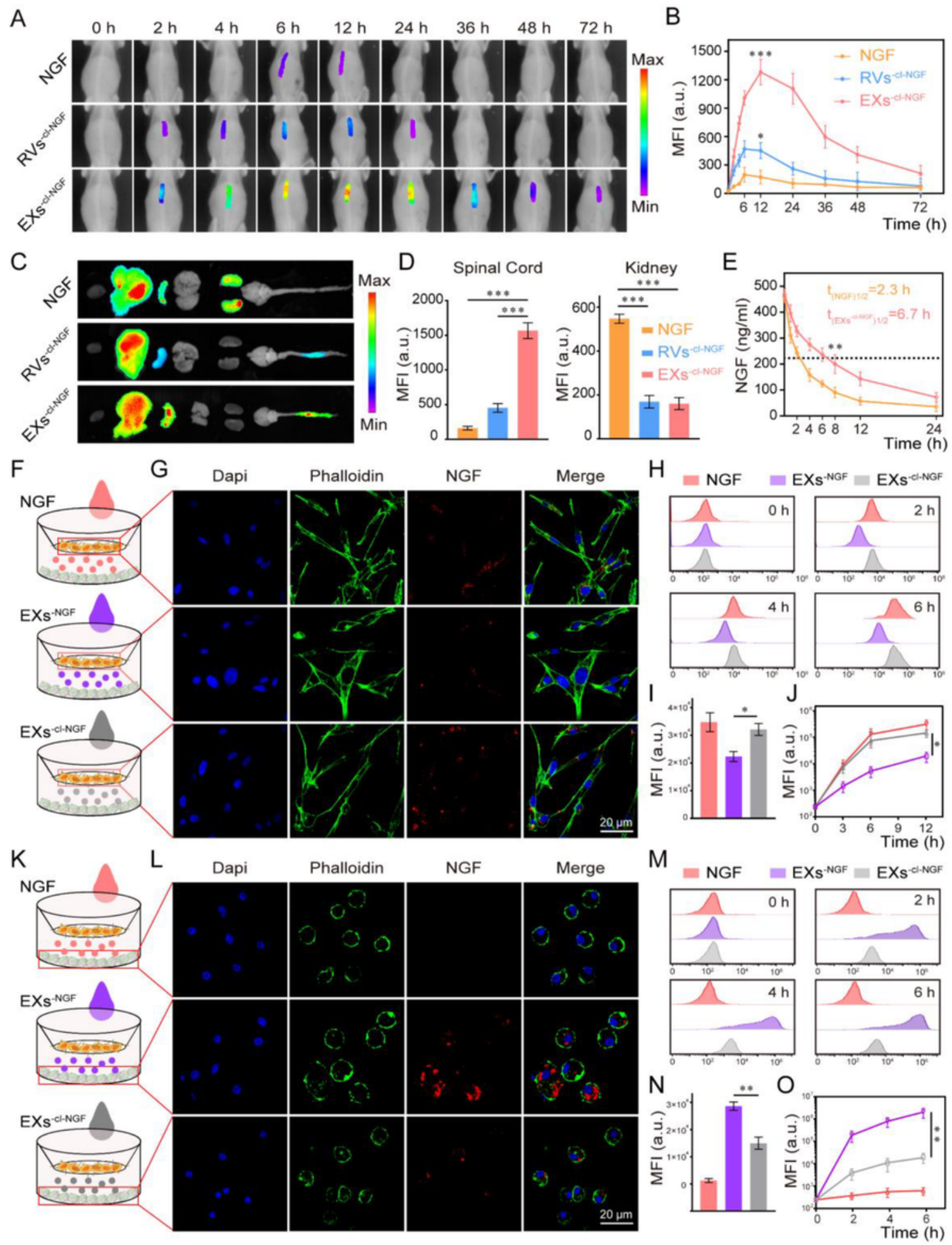

Figure 3 
The ability of EXs-cl-NGF to target sites of inflammation and release NGF. (A) NGF fluorescence imaging in injured spinal cord of different group mice after NGF, EXs-cl-NGF and RVs-cl-NGF injected by tail vein respectively, in different time points. (B) Fluorescence quantitative analysis of NGF inside injured spinal cord in different group mice at different time points. (C) The fluorescence imaging of the vital organs (heart, liver, spleen, lung and kidney) and spinal cord from different groups mice at 12 hours after tail vein injection. (D) Fluorescence quantitative analysis of spinal cord and kidney in different groups. (E) The concentration of NGF in plasma after injection of free NGF and EXs-cl-NGF at different times. $(F, K)$ Schematic diagram of the co-culture and the uptake of primary macrophages and PC12 cells in different groups. (G) CLSM images of the uptake of NGF in PC12 cells cultivated at the upper level of TranswellTM co-culture system. (H) Flow cytometry analysis of the uptake of NGF by PC12 cells cultivated at the upper level of TranswellTM co-culture system. (I) Fluorescence quantitative statistical analysis of Figure G. (J) Fluorescence quantitative statistical analysis of Figure $\mathrm{H}$. (L) CLSM images of the uptake of NGF in primary M1 macrophages cultivated at the lower level of TranswellTM co-culture system. (M) Flow cytometry analysis of the uptake of NGF by primary M1 macrophages cultivated at the lower level of TranswellTM co-culture system. (N) Fluorescence quantitative statistical analysis of Figure L. ( 0 ) Fluorescence quantitative statistical analysis of Figure M. Data presented the mean $\pm S D(n=6$ per group) ( ${ }^{\star} \mathrm{P}<0.05,{ }^{\star *} \mathrm{P}<0.01,{ }^{\star *} \mathrm{P}<0.001$ and $\mathrm{ns}$ : not significant) 

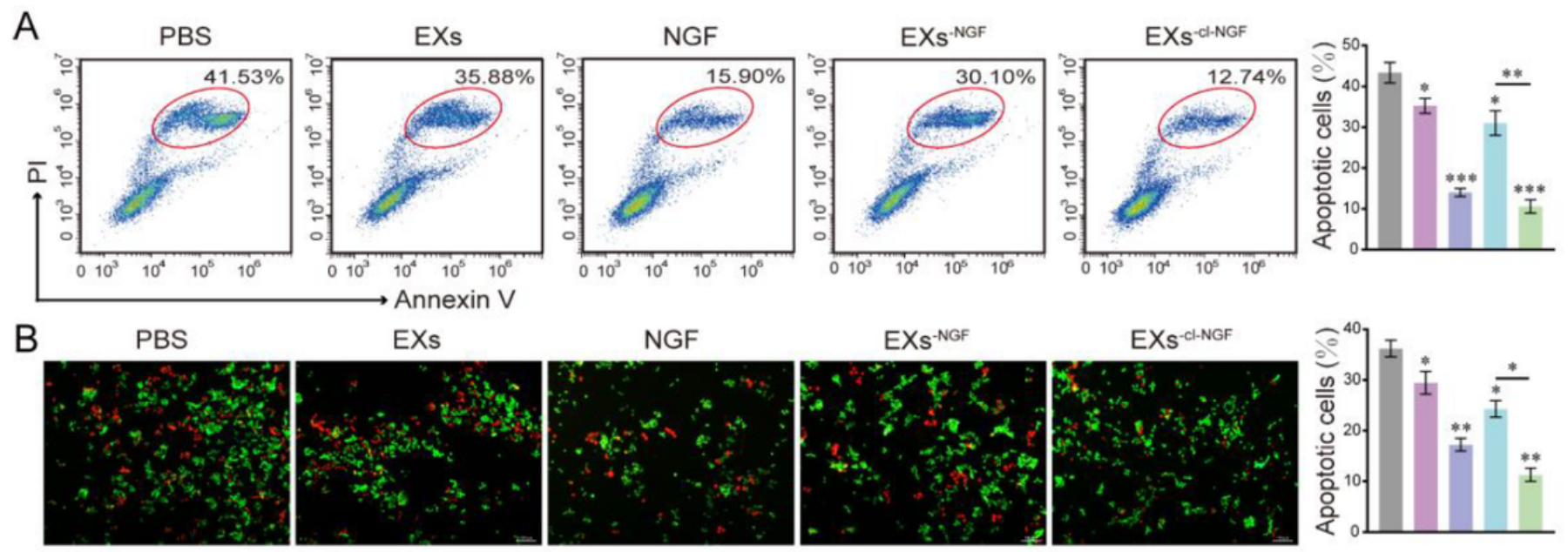

C
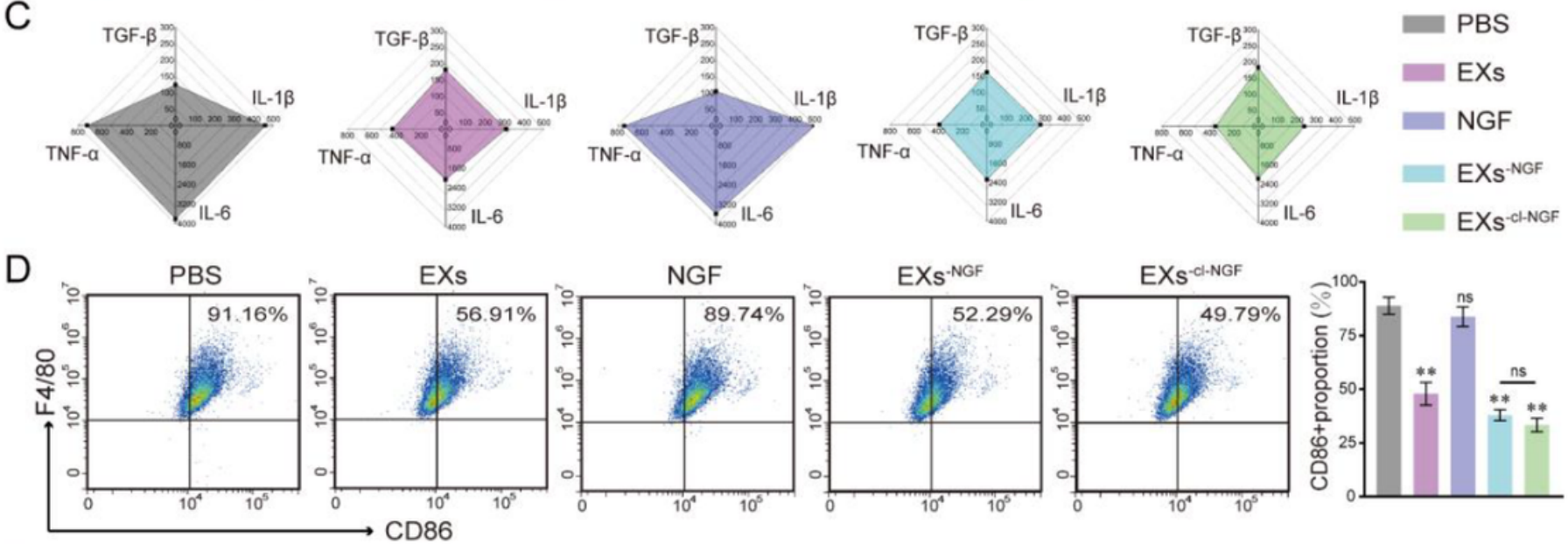

E
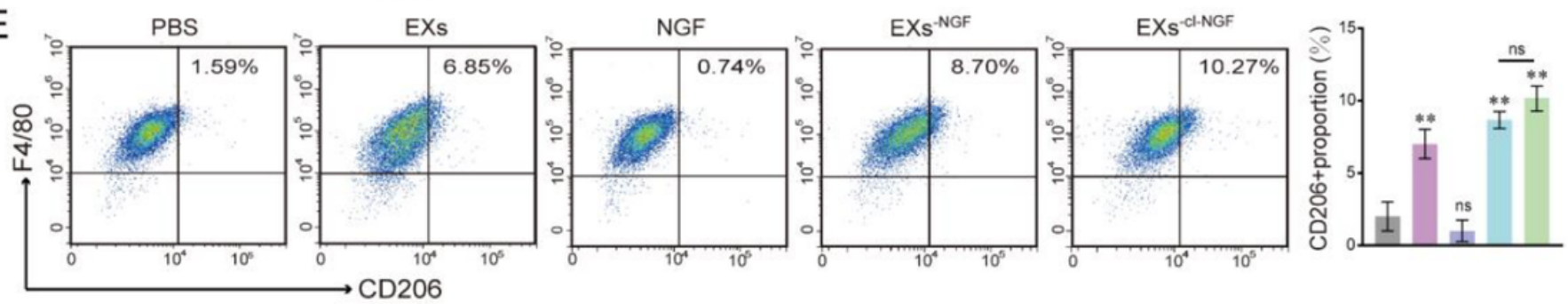

\section{Figure 4}

In vitro inflammation regulation and neuroprotective capabilities of EXs-cl-NGF. (A) Flow cytometry analysis of the neuroprotection of EXs-cl-NGF on PC12 cells cultivated at the upper level of TranswellTM co-culture system. (Numbers in the areas indicated percent late apoptotic cells) (B) Live-Dead cell staining to evaluate the neuroprotection of EXs-cl-NGF on PC12 cells cultivated at the upper level of TranswellTM co-culture system. (C) ELISA-based measurement of TGF- $\beta$, IL- $1 \beta$, IL-6, and TNFa $(\mathrm{pg} / \mathrm{mL})$ in supernatants prepared from the culture medium form different groups. (D) Flow cytometry analysis of primary M1 macrophages cultivated at the lower level of TranswellTM co-culture system. (Numbers indicated percent F4/80+, CD86+ cells). (E) Flow cytometry analysis of primary M2 macrophages cultivated at the lower level of TranswellTM co-culture system. (Numbers indicated percent F4/80+, CD206+ cells). * at the top of each group's statistical graph indicates the difference analysis with the PBS 
group. Data presented the mean $\pm S D$ ( $n=6$ per group) $(* P<0.05, * \star P<0.01, * \star \star P<0.001$ and ns: not significant)

A

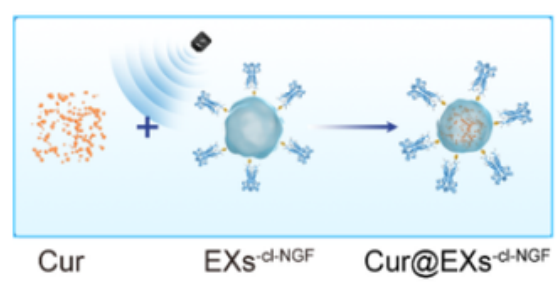

B

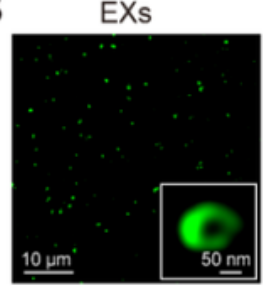

NGF

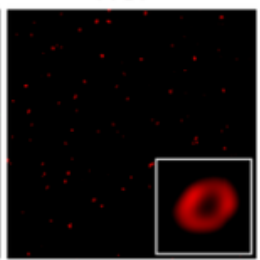

Cur

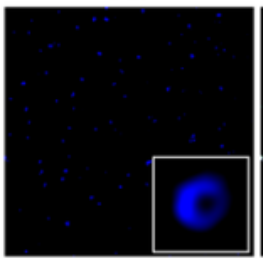

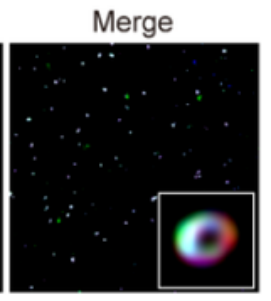

C

D PBS

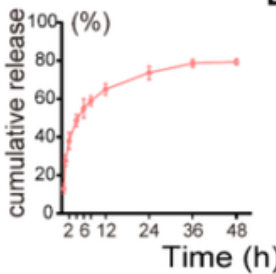

$\mathrm{E}$
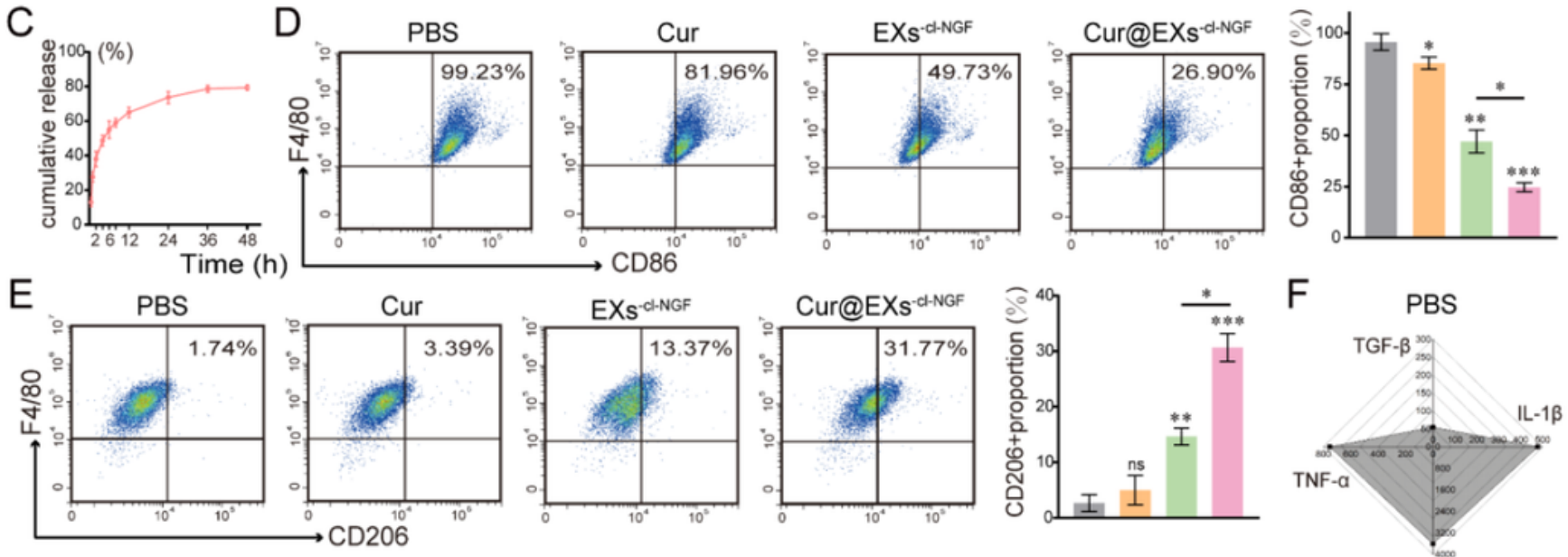

G

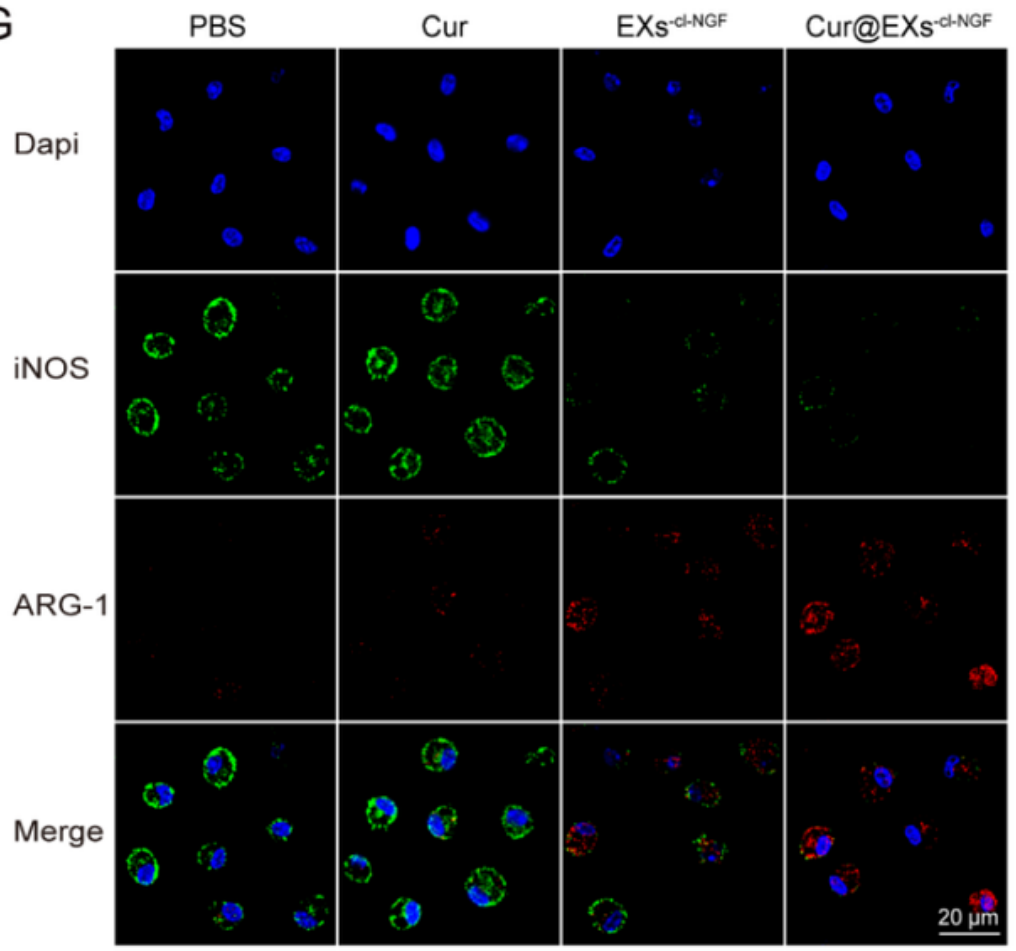

$\mathrm{H}$
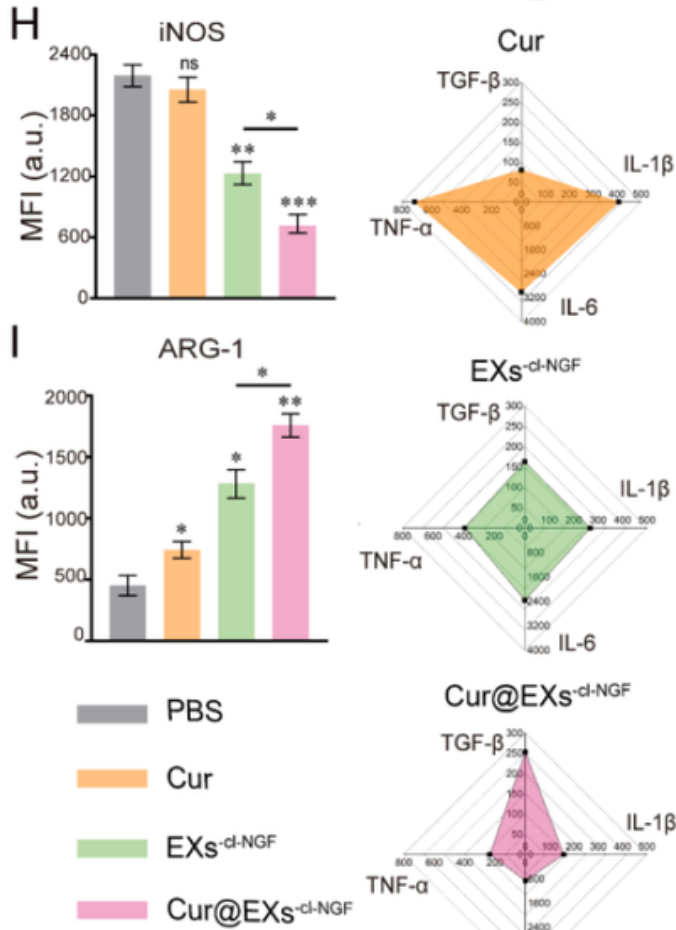

Cur@EXs'-l-NGF TGF- $\beta$,

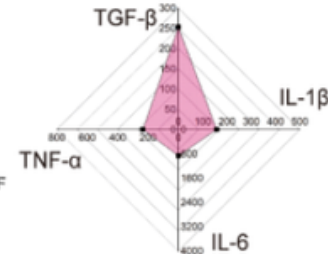

\section{Figure 5}

The inflammation regulation capabilities of Cur@EXs-cl-NGF. (A) Schematic diagram of Cur loading into EXs-cl-NGF. (B) CLSM images and the STED images (enlarged illustration in lower right corner) of Cur@EXs-cl-NGF, EXs (green), NGF (red) and Cur (blue). (C) In vitro Cur release of Cur@EXs-cl-NGF (D) 
Flow cytometry analysis of primary M1 macrophages cultivated at the lower level of TranswellTM coculture system. (Numbers indicated percent F4/80+, CD86+ cells). (E) Flow cytometry analysis of primary M2 macrophages cultivated at the lower level of TranswellTM co-culture system. (Numbers indicated percent F4/80+, CD206+ cells). (F) ELISA-based measurement of TGF- $\beta$, IL-1 $\beta, \mathrm{IL}-6$, and TNFa $(\mathrm{pg} / \mathrm{mL})$ in supernatants prepared from the culture medium. (G) Representative fluorescence images of the macrophage phenotypes. $(\mathrm{H})$ Fluorescence quantitative statistical analysis of iNOS of Figure G. (I) Fluorescence quantitative statistical analysis of ARG-1 of Figure G. * at the top of each group's statistical graph indicates the difference analysis with the PBS group. Data presented the mean \pm SD $(n=6$ per group) ( ${ }^{*}<0.05,{ }^{* *} \mathrm{P}<0.01,{ }^{* \star *} \mathrm{P}<0.001$ and $\mathrm{ns}$ : not significant)


E
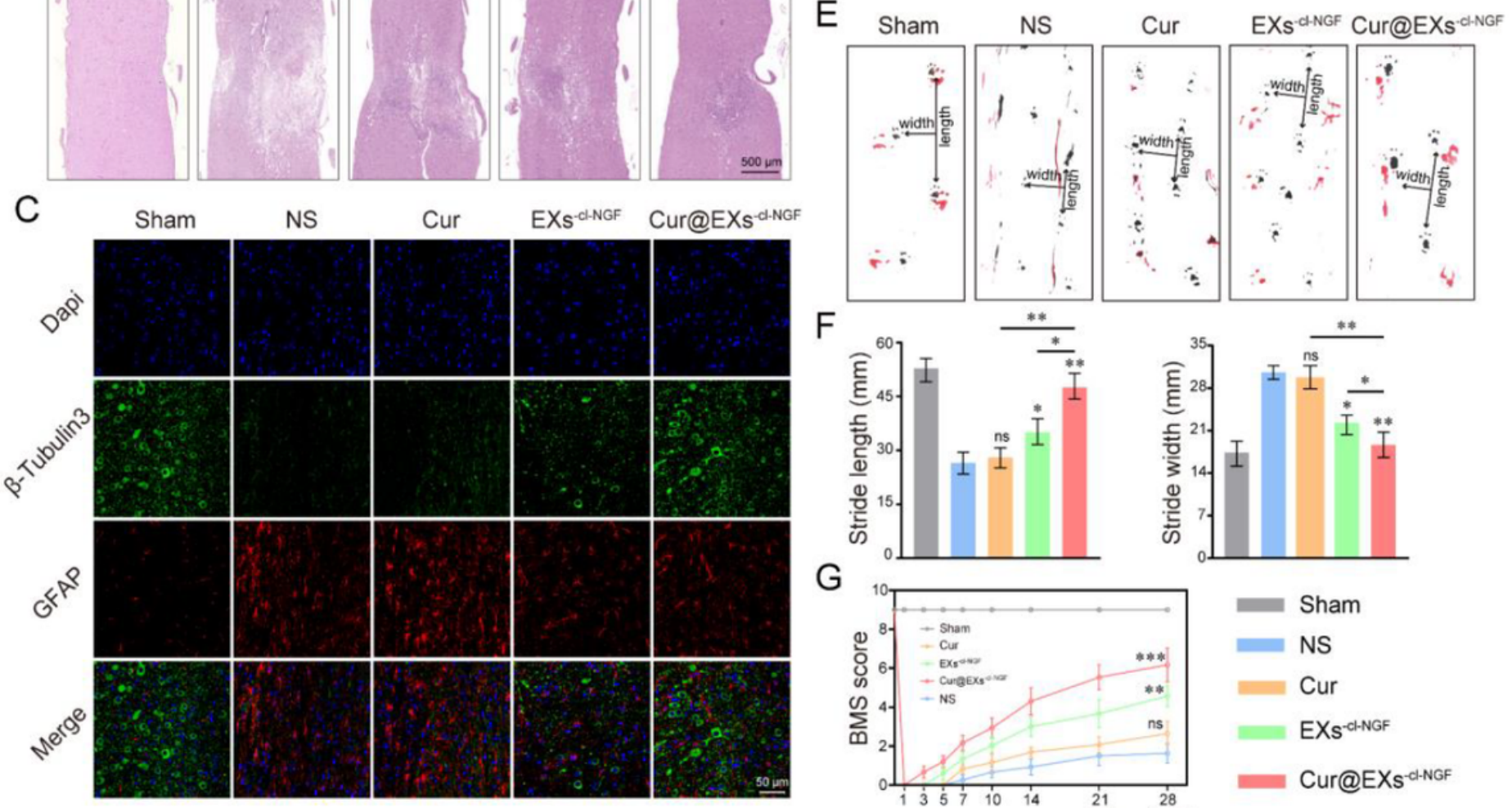

Cur


G

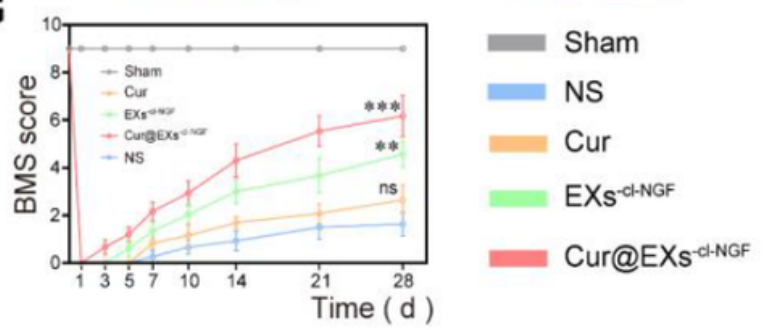

\section{Figure 6}

Cur@EXs-cl-NGF promotes the recovery of motor function after $\mathrm{SCl}$. (A) Ultrastructure of myelin sheaths from different groups determined by TEM. (B) H\&E stained images of spinal cord tissue from different groups. (C) Fluorescence images of spinal cord tissue, in which green channel represented neuronal axon tubulin (B3-Tubulin) and red channel represented astrocytes (GFAP). (D) Fluorescence quantitative statistical analysis of $\beta 3$-Tubulin and GFAP in figure $C$. (E) Representative images of footprint behavioral assays. (F) Quantitative analysis of stride length and stride width in footprint assays. (G) Mice motor 
functional recovery of different groups mice during treatment using BMS scores for statistical analysis. ( $n=12$ per group). * at the top of each group's statistical graph indicates the difference analysis with the NS group. Data presented the mean \pm SD $(n=6$ per group, except for data $G)\left({ }^{\star} P<0.05,{ }^{\star \star} P<0.01, \star \star \star P<\right.$ 0.001 and ns: not significant)

A
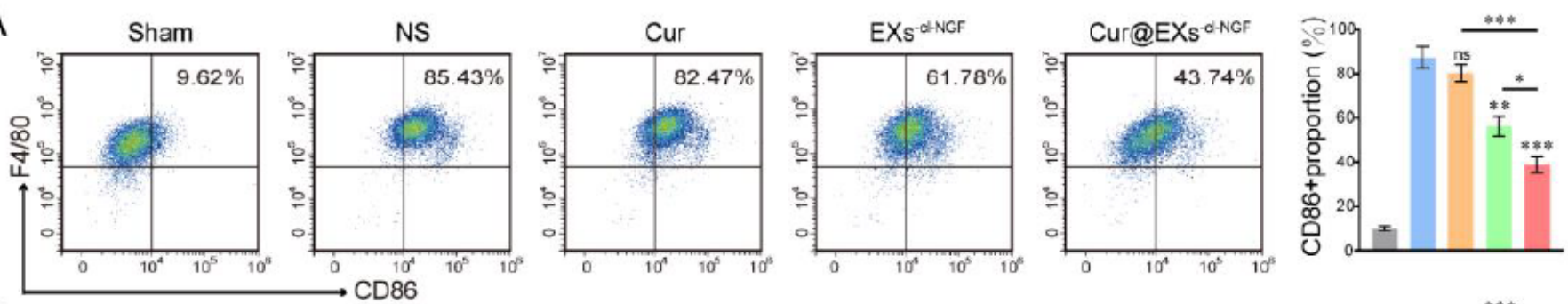

B
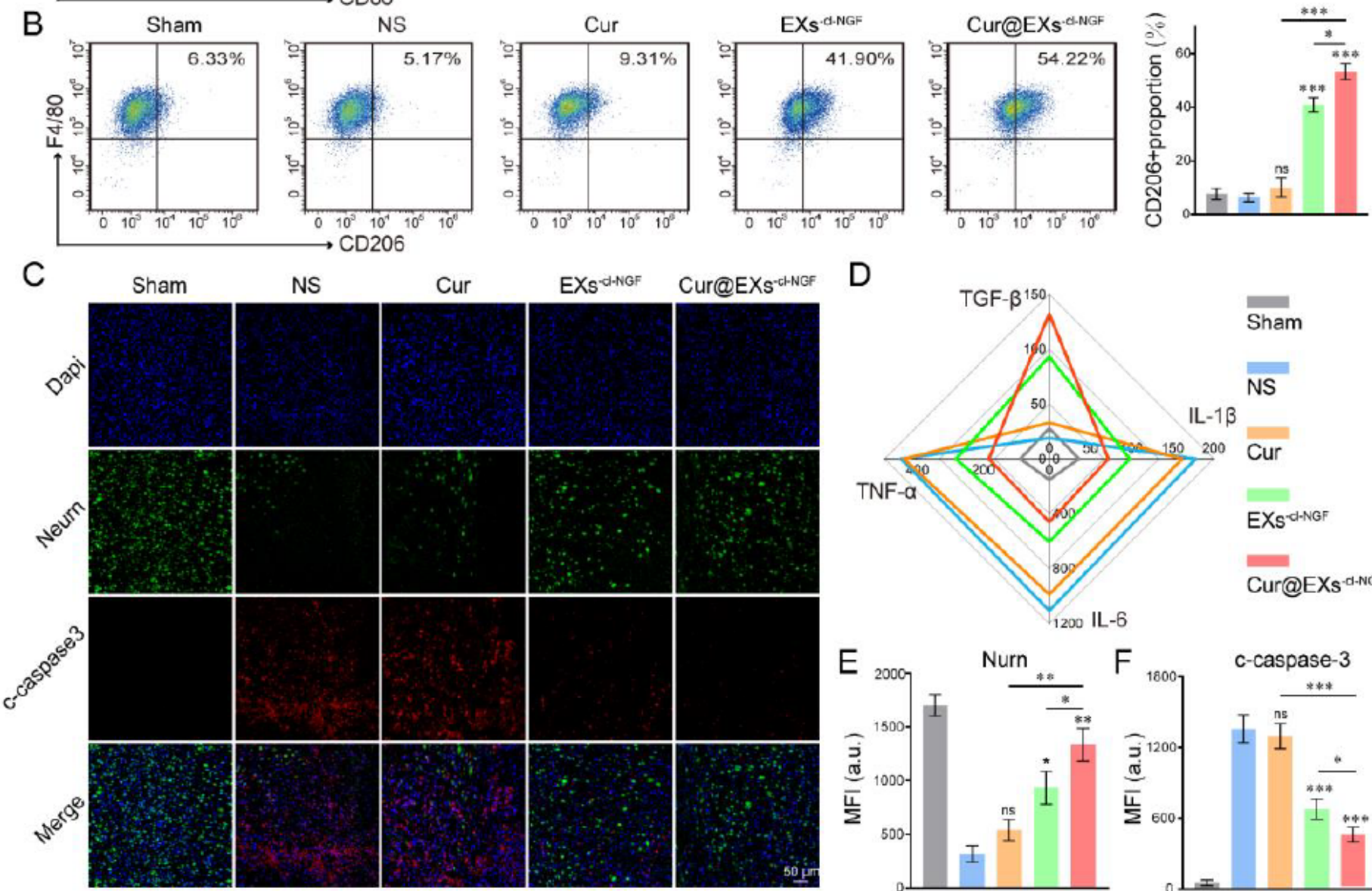

NS
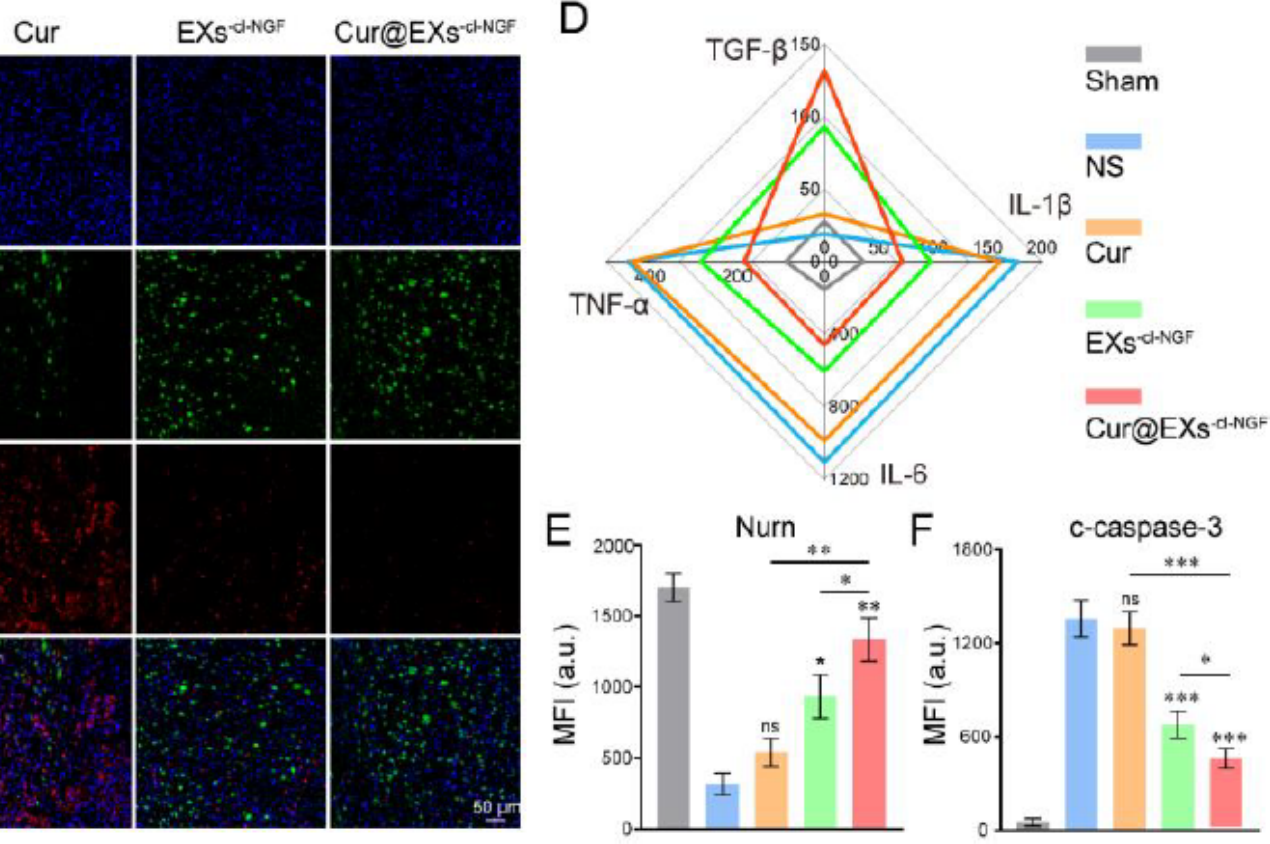

\section{Figure 7}

The role of Cur@EXs-cl-NGF in the early stages of injury. (A) Flow cytometry analysis of the ratio of M1 microglia/macrophages to total microglia/macrophages. (Numbers indicated percent F4/80+, CD86+ cells). (B) Flow cytometry analysis of the ratio of M2 microglia/macrophages to total microglia/macrophages. (Numbers indicated percent F4/80+, CD206+ cells). (C) Fluorescence images of spinal cord tissue, in which green channel represented neurons (NeuN) and red channel represented apoptosis marker protein (c-caspase3). (D) ELISA-based detection of pro-inflammatory factors (TNF-a IIL$1 \beta \mathbb{Z I L}-6)$ and anti-inflammatory factors (TGF- $\beta$ ) in the injured spinal cord tissue. (E, F) Fluorescence quantitative statistical analysis of Nurn and c-caspase 3 in figure C. * at the top of each group's statistical 
graph indicates the difference analysis with the NS group. Data presented the mean \pm SD $(n=6$ per group) $\left({ }^{*} \mathrm{P}<0.05,{ }^{* *} \mathrm{P}<0.01,{ }^{* \star *} \mathrm{P}<0.001\right.$ and ns: not significant)

\section{Supplementary Files}

This is a list of supplementary files associated with this preprint. Click to download.

- Graphicalabstractimage.png

- Supplementarymaterial.docx 